\title{
RECENT ADVANCES IN NEURORADIOLOGY
}

\author{
G. F. Swann, M.R.C.P., D.M.R.D. \\ Consultant Radiologist, Westminster Hospital, London, S.W.I, and West End Hospital for Neurology \\ and Neurosurgery, London, W.I
}

A COMPLETE review of all the neuroradiological literature has not been attempted in this account. Detailed consideration has been given to those aspects which have become more important as the result of developments in other fields; for example, improved anæsthesia with hypothermia has made possible more leisurely surgical treatment of arteriosclerotic vessels and the Spitz-Holter valve has made the study of infantile hydrocephalus more rewarding.

Improvements in technique follow two main lines; firstly to produce better radiographs of normal and abnormal anatomy, particularly of anatomical regions hitherto considered inaccessible to the radiologist, and secondly to reduce the hazards to the patient when obtaining these X-rays.

To X-ray inadequately is almost as bad as to $\mathrm{X}$-ray unnecessarily. Frequently patients come to neurological centres with poor-quality $\mathrm{X}$-rays of special investigations which have to be repeated. The occasional neuroradiologist should be discouraged unless he has the necessary apparatus and a sound training.

Where possible, personal experience with the techniques described is given.

Although radiology plays an important part in stereotactic surgery it has not been included in this account.

\section{Apparatus \\ Mimer}

This new X-ray diagnostic unit could be described, without being fanciful, as the direct descendant of the original skull unit invented by Professor Lysholm of the Serafimer Hospital and Georg Schonander of the firm Schonander Jarn in I93 I, because the new unit has been invented by Professor Erik Lindgren of the same hospital and department and Georg Fredzell of the firm Elema Schonander A.B. (Fredzell and Lindgren, 1960). The apparatus consists of a wall-floor stand supporting a cantilever (Figs. I and 2). From this a descending limb, which can move on a vertical axis, articulates with an ascending limb, which can move on a horizontal axis. At the upper end of the ascending limb is placed an X-ray tube. These two limbs form a $\mathrm{V}$ in the neutral position and their point of articulation lies at the level of the plane of the film, which is carried on yet another limb mounted lower down on the wall-floor column. The object table is basically the same as that in the Lysholm skull unit. The limb supporting the object table is disposed horizontally and can be swung out of the way so that a serial film changer or mobile trolley can be wheeled under the tube. A fluoroscope unit with a cassette holder can be attached to the tube so that fluoroscopy and radiography can be carried out around the patient in any plane. Movements of the descending limb in a vertical plane and of the ascending limb in a horizontal plane can place the focal spot of the $\mathrm{X}$-ray tube anywhere on a spherical surface with a radius of $80 \mathrm{~cm}$. A film-focus distance of between 70 and $90 \mathrm{~cm}$. is obtained by raising or lowering the object table. The tube mounting can be decentralized if necessary. Centring is obtained by a light pointer. All movements are motor assisted at two speeds. The latest model has available a tomographic attachment and facilities to attach an image intensifier.

Not only has this unit facilitated neuroradiological procedures, but, if it is used with a mobile trolley and an image intensifier, transfemoral and transbrachial cerebral arteriography are simplified because of the ease with which the catheter can be screened into the appropriate place. This apparatus is ideal for the investigation of the seriously injured patient who must not be moved. Tomographic studies of facial injuries are also possible without moving the patient.

\section{Myelographic Attachments to Supertilt Table}

Myelograms are arduous examinations, particularly for the radiologist. The supertilt table (Messrs. Watson \& Sons) has attachments designed to facilitate myelography. An account of experience with these attachments has been published (Swann, 1960). The prototype described in this paper has been improved and the final design agreed upon (Figs. 3 and 4 ). Basically the apparatus consists of two $\mathrm{X}$-ray tubes and two fluoroscopic units. Both fluoroscopic units are 


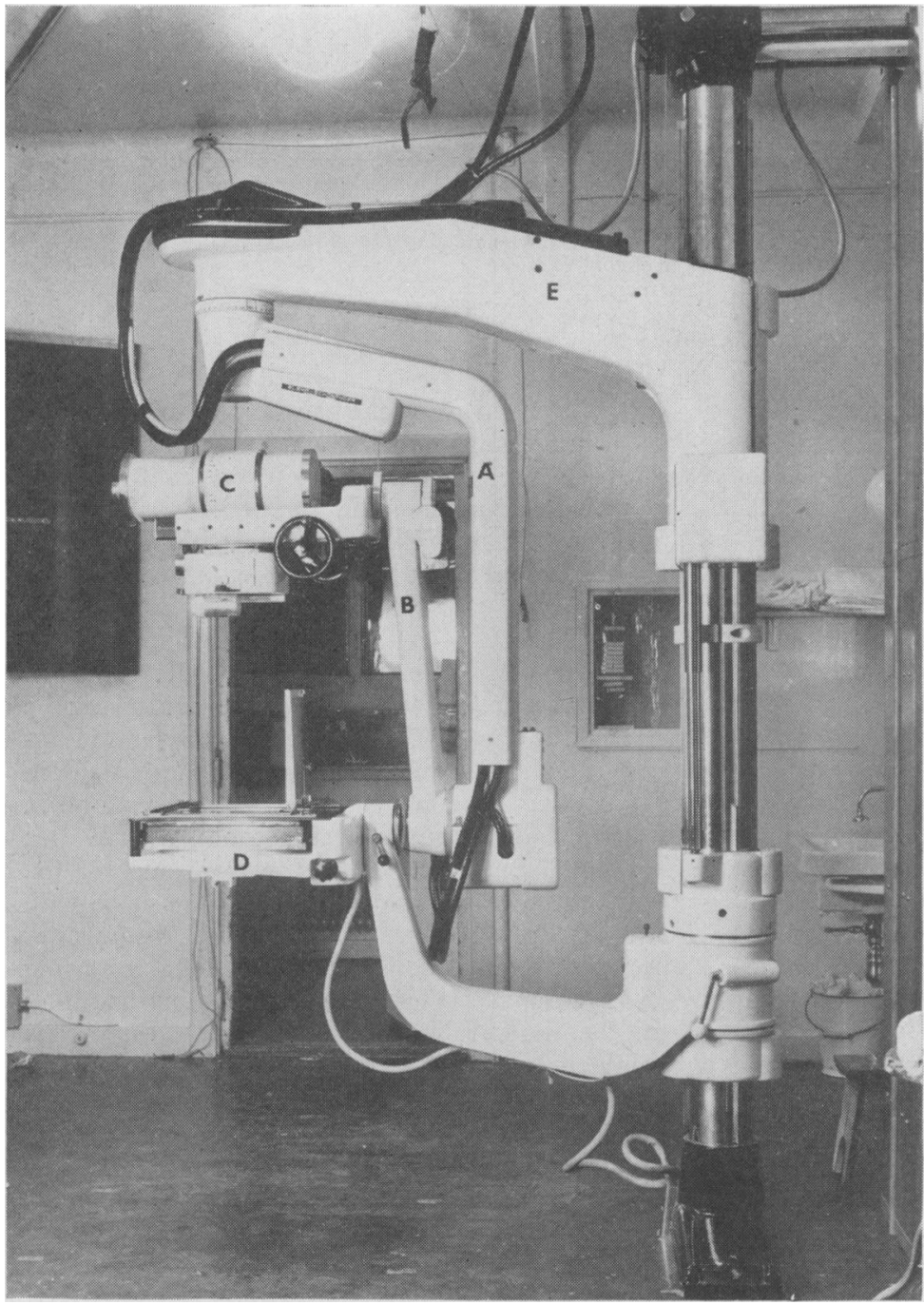

FIG. I.-Mimer. The apparatus is here shown in the neutral position: (A) Is the descending limb; (B) the ascending limb; (C) the X-ray tube; (D) the object table; (E) the cantilever arm. All these are mounted upon the floor-ceiling column.

capable of rapid radiography. All these units are linked and their movements are motor assisted. Coupling of each X-ray tube with a screening unit is arranged to cover the lateral, postero-anterior and antero-posterior aspects of the spinal theca. The linked X-ray tubes are automatically centred at the same point, enabling rapid change to be made from antero-posterior to lateral screening and the reverse. Thus the contrast medium can be viewed in two planes and accurately placed and maintained by use of tilt in the lordoses and kyphoses of the spinal column. A two-piece nylon harness allows the patient to be tilted $90^{\circ}$ cach way in relative comfort. The screening and radiographic unit for lateral views can be moved not only in the long axis of the patient but also supero-inferiorly and thus it is able to follow the curves of the spine in the lateral view. The lateral $\mathrm{X}$-ray tube is fitted with an adjustable slit diaphragm. Quick-release devices on the harness enable the patient to be screened prone, supine, and oblique in any degree of tilt (Swann, 1961).

\section{Harness for Encephalography}

A new harness for encephalography has been devised in the department of Docent Wickbom in the Salgrenska Hospital, Goteborg, Sweden (Lanner, 196I). This is a sack-like canvas bag, with apertures for the legs, in which the patient is placed (Fig. 5). There is an aperture for the head and another in the region of the lumbar spine for lumbar puncture. From the shoulder regions the patient is maintained in the upright position by two cords fixed via metal rings to the canvas 
Fig. 2.-Mimer. The object table has been swung out of the way and lies behind the floorceiling column. The descending $\operatorname{limb}(A)$ has been rotated through $180^{\circ}$; the ascending limb (B) through $25^{\circ}$ so that the tube $(C)$ is in the halfaxial position in relation to the antero-posterior film carrier $(F)$ of the rapid serial film changer (D).

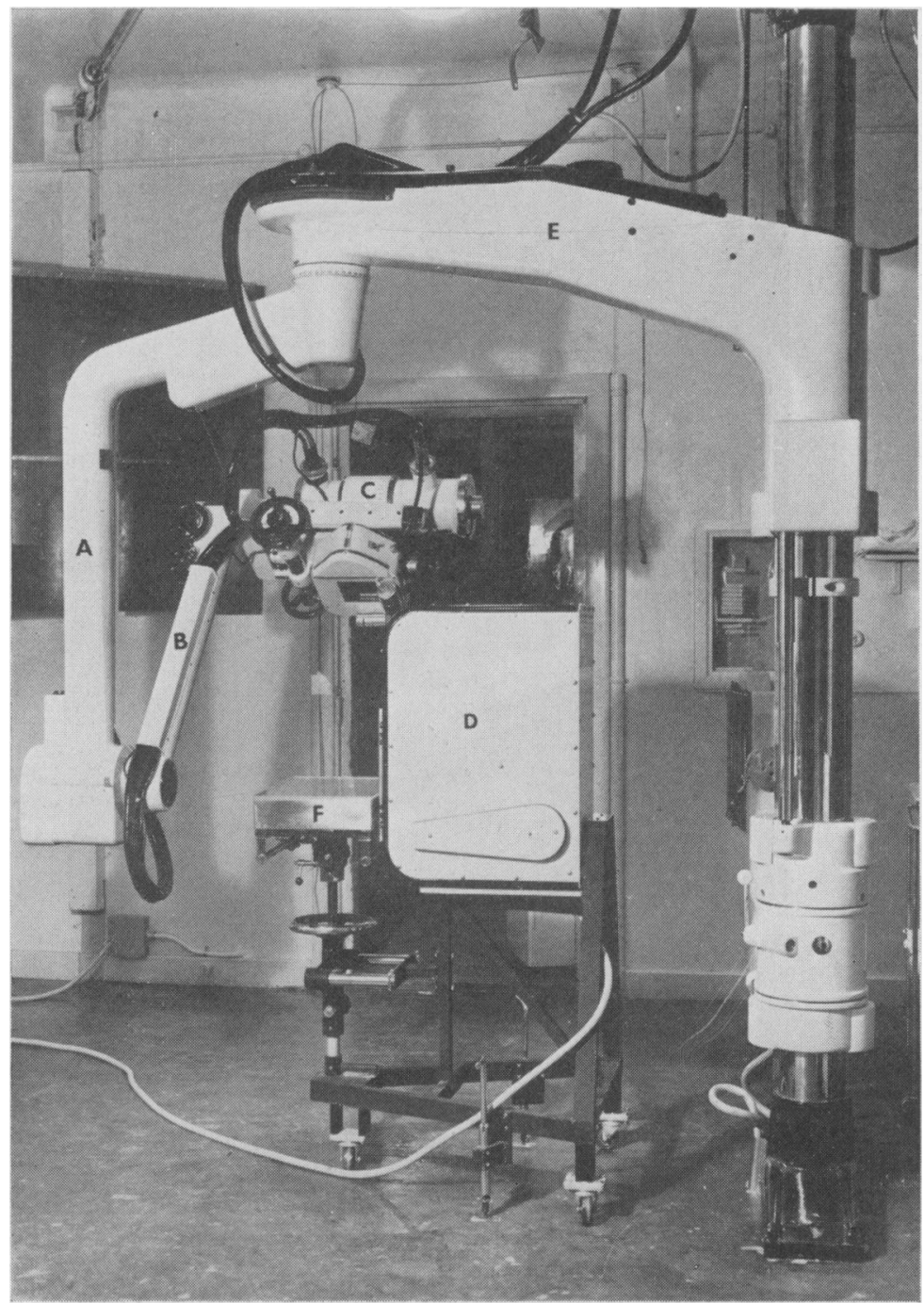

portion. These are joined to a cross-bar and from here to a pulley-ceiling suspension which can be controlled from an adjacent wall. Thus the recumbent and anæsthetized patient can be easily and gently raised into the sitting position.

In the latest model (not in the present illustration) the head halter is spliced into the support which runs from patient to ceiling suspension. Thus the patient can be accurately positioned and the skull unit can be swung into position, the patient being independently maintained in the correct posture without being attached to the $\mathrm{X}$-ray unit. This is an ideal harness in which to perform encephalography, particularly in the anæsthetized patient.

\section{Cerebral Pneumography}

Anatomy of the Subarachnoid Cisterns

The anatomy of the cisterns has been described most recently by Liliequist (I959a), who classifies them into supratentorial and infratentorial and those in the region of the tentorial hiatus. The exact form of the ambient cistern has been shown, particularly the relationship of the wings to the body of the cistern. Because of the disposition of the wings round the back of the pulvinar of the thalamus they are seen best in the lateral view, being upright crescent-shaped shadows. In hydrocephalus they are rotated backwards by the large ventricles so that their concave surfaces look superiorly. They may be displaced and rendered 


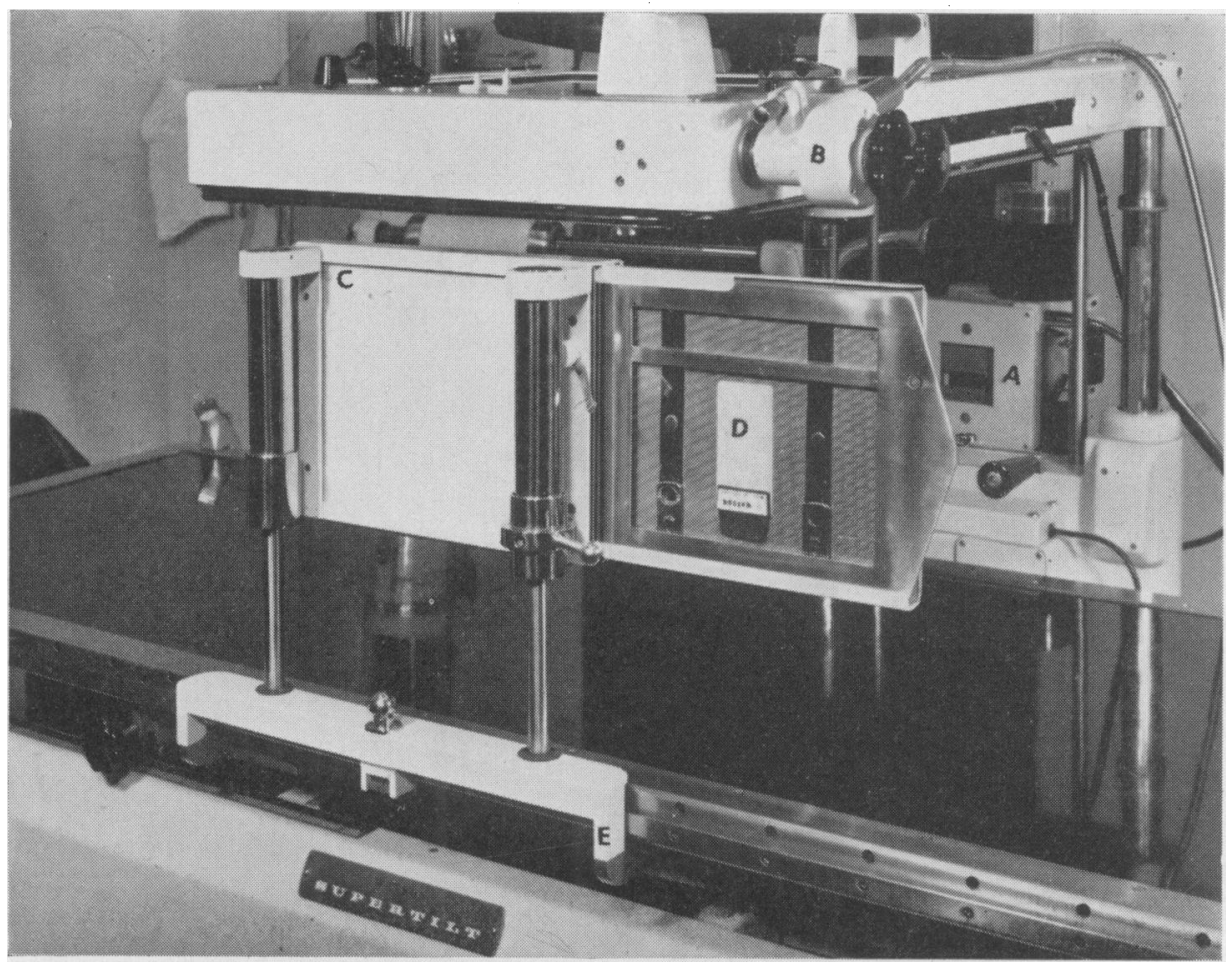

FIG. 3.-Myelographic attachments shown in detail: (A) The lateral tube with slit diaphragm; (B) the controls for the slit diaphragm; (C) lateral fluoroscopic unit; (D) lateral radiographic unit with 10 in. $x 8$ in. film in position with the whole unit raised above the table top; (E) attachment to Potter-Bucky grid carrier.

asymmetrical by intracranial masses (Schmitz, 1959; Klaus, 1958).

A new encephalographic sign of pressure hydrocephalus has been described (Schechter, Bull and Carey, 1958). This consists of dilatation of the wings of the ambient cisterns (which may be unilateral or bilateral), of the sulci corporis callosi (which may look rather like ' rabbits ears' in antero-posterior view) and of the cingulate sulci. All cases were the result of posterior fossa.spaceoccupying lesions or aqueduct stenosis (a case has been seen, however, with a colloid cyst of the third ventricle; Fig. 6). It is suggested by these authors that the pathogenesis of these appearances was an intermittent hydrocephalus associated with brain atrophy.

Liliequist identified the crural cisterns around the cerebral peduncles and contrasted them with the olfactory sulci. Both these paired spaces are seen in antero-posterior view and appear as crescent-shaped shadows, the olfactory sulci being more upright and medially placed (Fig. 7). The intercrural cistern (interpeduncular cistern) is shown to be a midline structure connected to the crural cisterns and lying between the cerebral peduncles.

The cavum veli interpositi is a midline space lying above the roof of the third ventricle communicating behind with the quadrigeminal plate and ambient cisterns and not extending anteriorly beyond the Foramen of Munro. It appears as a fan-shaped structure lying between the lateral ventricles in antero-posterior view. It is of somewhat academic interest to differentiate it from a cavum vergæ as this does not exist without a cavum septum pellucidum and merges with it. This, in turn, extends anteriorly to beyond the Foramen of Munro and this provides the differentiation (Zellweger and Van Epps, 1960).

\section{Pontine Angle Tumours}

Liliequist (1959b), in a paper on a series of 64 pontine angle tumours (of which 50 were acoustic neuromas), re-emphasizes the difficulties in the diagnosis of small tumours in this region. This problem has been discussed by other authors, in 

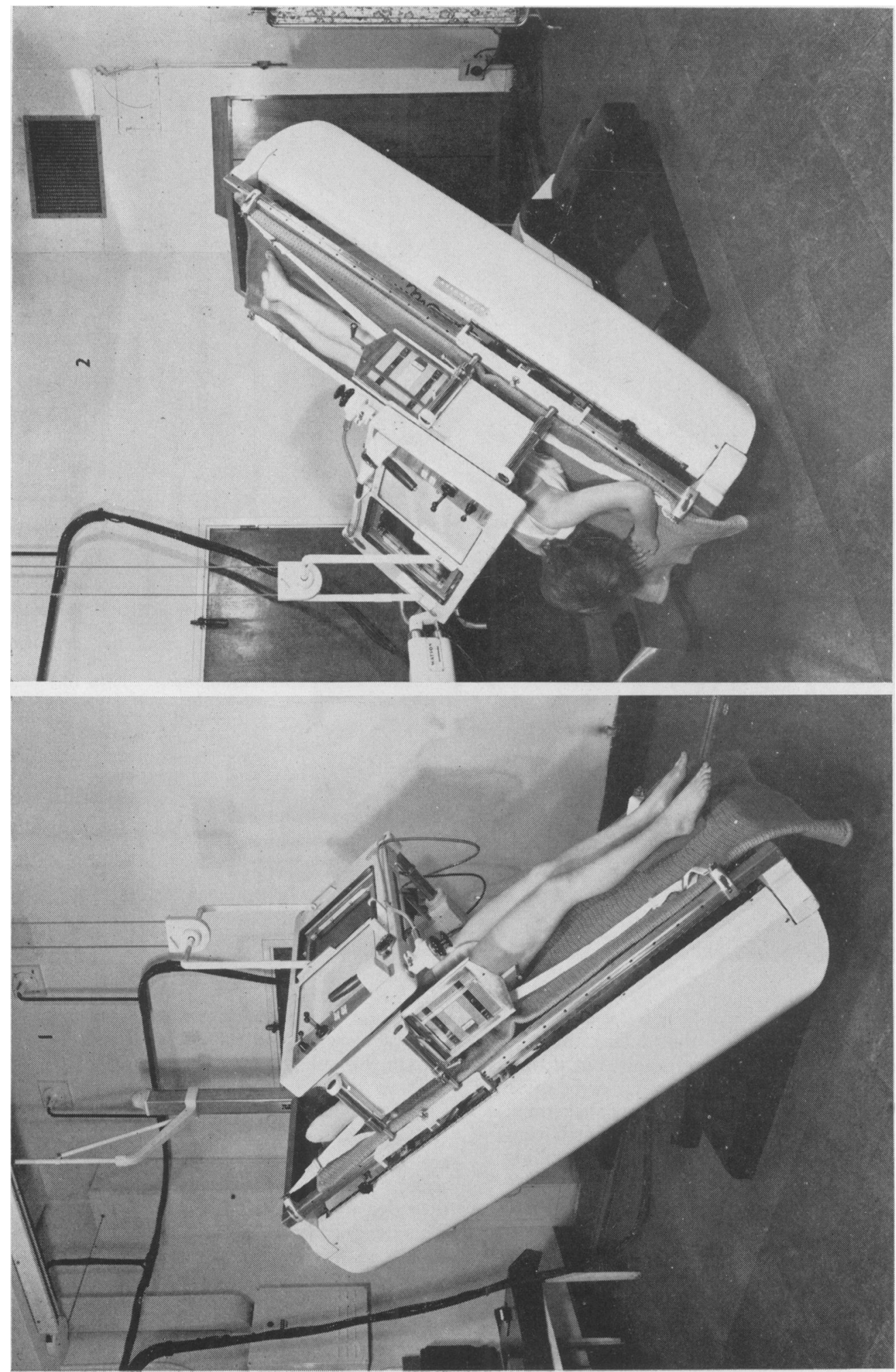

원

ส․ㅇํ

so

范荡

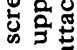

푼

$\underset{2}{2}$

这

F:

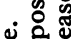

西要

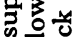

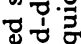

政

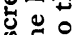

00 눙

웡

节学

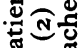

a.

政

다응

क

3.음

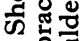

$\Xi \xi$

둥

果要

喓

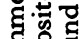

응응 है

氙

过

so.

웡항

s.

广象

宦 


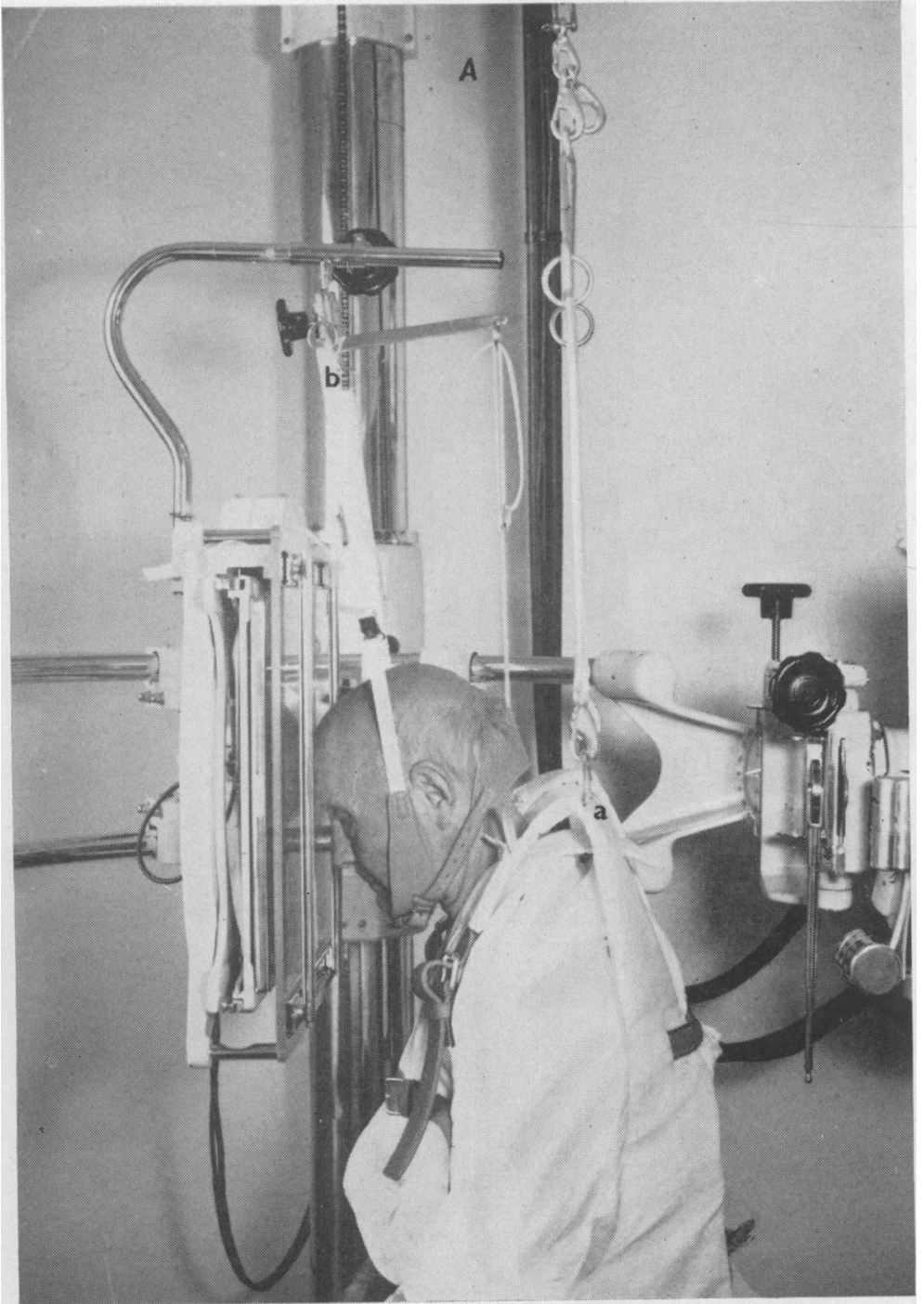

FIG. 5.-The encephalographic harness (a) is seen in the lateral view. The head halter (b) is seen attached in the conventional way to the skull unit. In the latest model of the harness this halter is 'spliced" into the ceiling suspension of the harness. Thus the patient can be positioned independently of the X-ray apparatus, facilitating the positioning of the X-ray tube and enabling an anæsthetized patient to be efficiently supported.

particular Lindgren (1957). When the cerebellopontine angle cistern fills the deformities the filling defects are usually recognizable, but if they do not fill they may contain a tumour or be quite normal and fill on a subsequent encephalogram. Small tumours will not displace the fourth ventricle or aqueduct but will give rise to filling defects and distortions of the brain stem resulting in asymmetry of the ponto-cerebellar and ambient cisterns.

\section{Brain Herniations}

Any account of advances in neuroradiology should take note of the paper published in $195^{6}$ by Azambuja, Lindgren and Sjögren, which describes the morbid anatomy, angiography and cerebral pneumography of up-and-down herniation through the tentorial hiatus.

\section{Congenital Abnormalities}

Congenital Defects of the Tentorium. Two cases are described (Liliequist, Tovi and Schisano, I960). A huge cisterna magna was present which extended above the usual confines of the tentorium. This developmental defect may be associated with defects in the occipital bone (which may have a sclerotic margin), and anomalies of the straight sinus.

Arnold Chiari Malformation. The encephalographic manifestations of this anomaly and its 


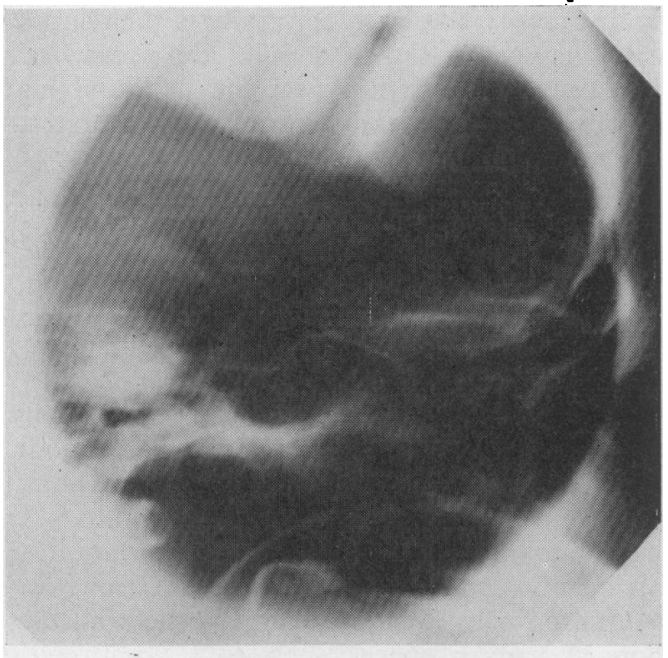

A

FIG. 6.-(A) Lateral view of anterior end of third ventricle shown at encephalography, using autotomography. There is a rounded filling defect in the roof of the third ventricle, behind the foramen of Munro. (B) An erect postero-anterior view showing the dilated sulci corporis callosi. A colloid cyst of the third ventricle was discovered at operation.

differentiation from herniated cerebellar tonsils due to space-occupying lesions is discussed (Liliequist, 1960). In the Arnold Chiari anomaly the air usually enters the foramen of Magendie outlining the abnormal fourth ventricle which is narrow and elongated, showing the foramen of Magendie to be below the foramen magnum. The cerebellar tonsils are closely applied to the medulla; they are pointed and triangular in shape and also lie below the level of the foramen magnum (Fig. 8). Immediately below them there is a bulge on the posterior surface of the medulla. The cervical part of the cisterna magna often contains some air. In contra-distinction, herniated cerebellar tonsils due to space-occupying lesions are round and plump and fill the cervical part of the cisterna magna. Frequently no air enters the foramen of Magendie and a posterior bulge on the medulla is not seen.

A classification of the types of Arnold Chiari malformation is given, the simplest type showing only tonsillar herniation, the second with both the fourth ventricle and the tonsils below the foramen magnum, and the most gross with a cervical spina bifida cystica, the sac containing most of the cerebellum. It is claimed that encephalography will show these appearances more consistently than ventriculography. It must be noted that only

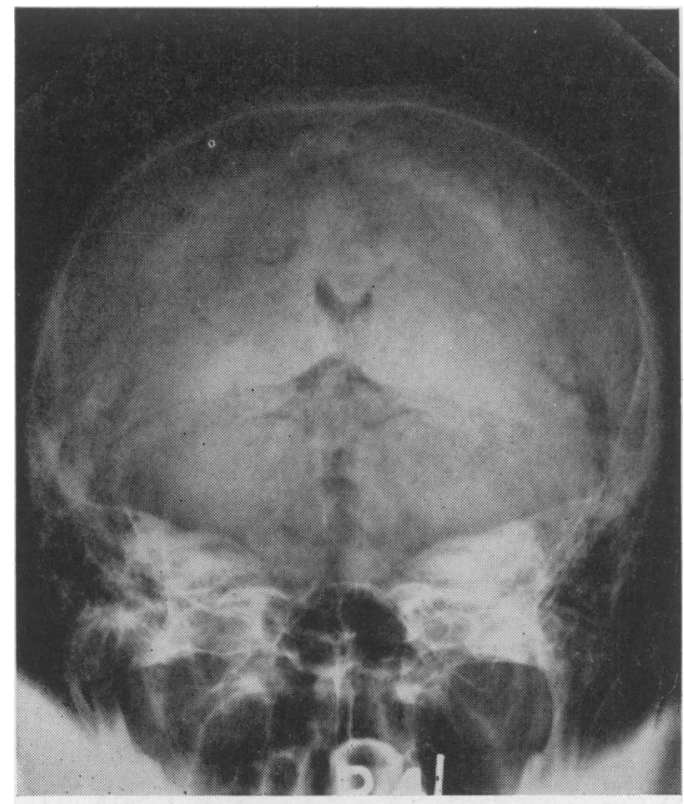

B

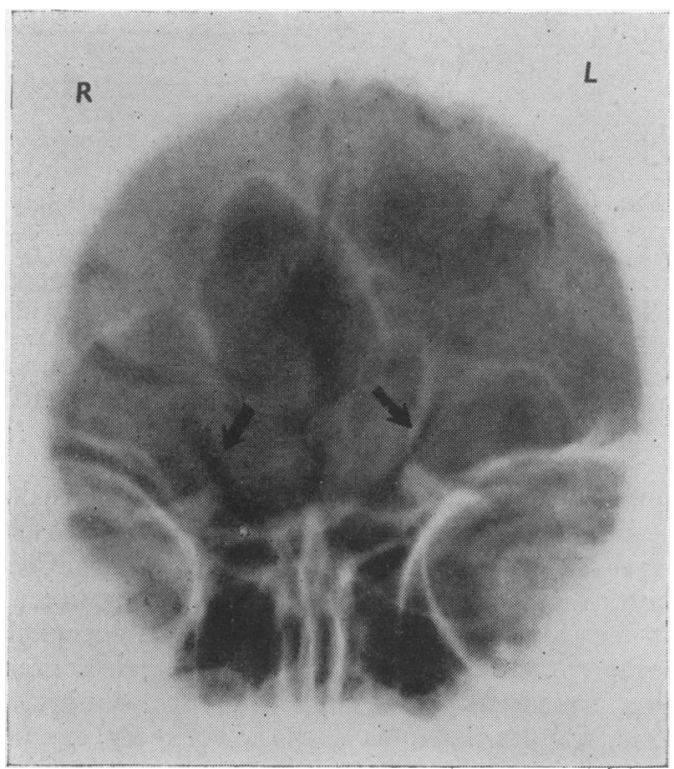

FIG. 7.-The olfactory sulci have been marked with arrows. The left is displaced laterally and narrowed. The right is less displaced. They surround a pituitary tumour. 

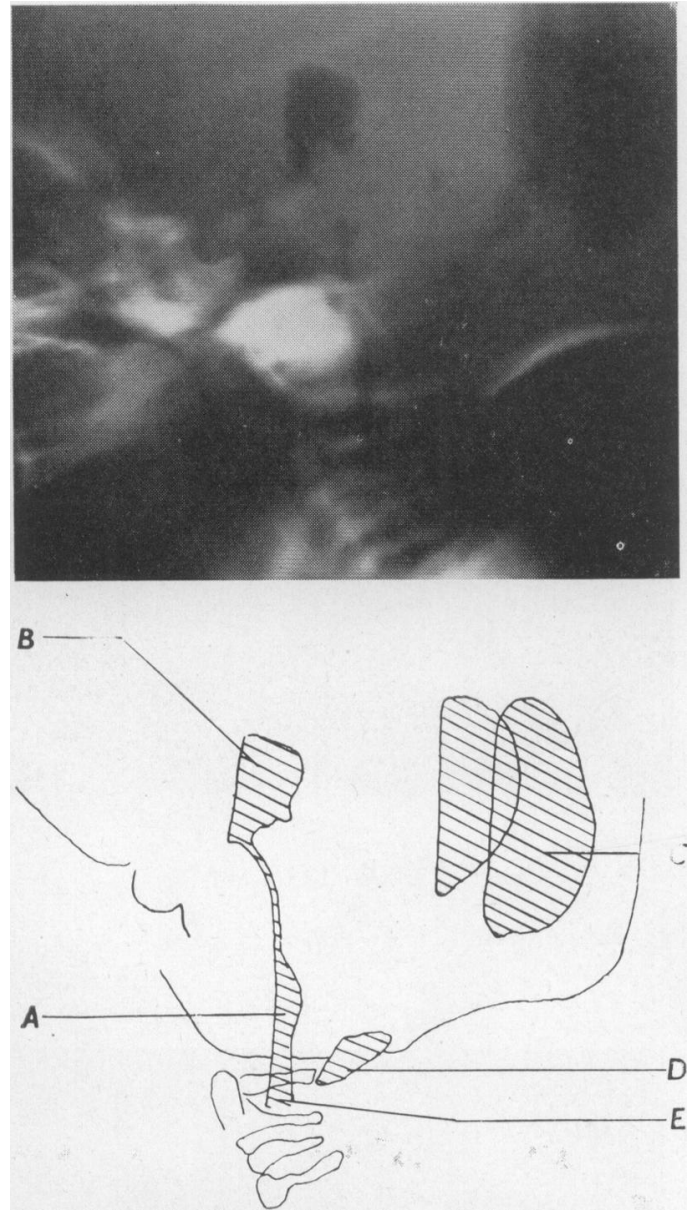

Fig. 8.-Arnold Chiari malformation demonstrated at ventriculography, using autotomographic technique. The shape of the fourth ventricle $(A)$ is long and narrow and the foramen of Magendie (E) lies below the foramen magnum. The triangularshaped cerebellar tonsils can be seen and there is air in the cervical part of the cisterna magna (D). The third ventricle (B) and lateral ventricle (C) contain air.

one of the cases in this series showed hydrocephalus. A personal series of egses with this malformation have presented with hydrocephalus after repair of the lumbar spina bifida cystica. In these cases both lumbar and cisternal encephalography were not feasible. In air ventriculograms performed on such cases using the technique of Ziedses des Palantes (1950), not only can the fourth ventricle be shown but air can be manipulated into the cisterna magna and the cervical subarachnoid space.

En passant, Liliequist makes the important observation that, if air is injected subdurally via the lumbar route, cerebellar tonsillar herniation may be caused; similarly, deformity of the pontine cistern may occur, the cerebellum and brain stem being displaced anteriorly by the subdural air. Distortion of the lateral ventricles by supratentorial subdural air is also recorded.

\section{The Use of the Image Intensifier in Cerebral Pneumography}

The value of this technique is common to all branches of radiology. Image intensifiers are beginning to be used routinely in several neuroradiological investigations. and in stereotactic surgery (Donaldson and Gillingham, 1960). Visual control of ventricular and cisternal filling during encephalography saves time and adds precision to the examination. It may be combined with a radiographic spot-film technique or with ciné-fluorography and occasionally the transient filling of narrow spaces such as a compressed chiasmatic cistern or third ventricle may be recorded by these methods but would have been less easy to demonstrate by conventional radiography. Similarly, somersault ventriculography (Ziedses des Palantes, I950) on babies may be watched, using a suitable image intensifier, and the diagnosis made more easily. Positive contrast ventriculography has always been a somewhat unsatisfactory method of examination because of the difficulties of visualizing a large space using a small volume of heavy, opaque medium. The extra visibility at low radiation dose which may be obtained with a large image intensifier goes some way to solving this problem (Du Boulay, I96r).

\section{Autotomography}

This technique was first described by Vallebona in I93 I and later by Ziedses des Palantes in 1950. It was called stratifigraphy and modified stratifigraphy by Ziedses des Palantes in 1956. The name autotomography was coined (Swann, 1957) for this manœuvre because $(a)$ it is descriptive, i.e. the patient can produce the effect by voluntary movements of the head, and $(b)$ the effect simulates tomography. It is a technique which may be used during cerebral pneumography to examine in the lateral view the median ventricles, the median subarachnoid cisterns of the brain and other midline structures. Vallebona caused the patient to turn the head to one side during the exposure of the lateral view of the skull. He gave no radiographic factors nor the speed or amplitude of the head movements. Ziedses des Palantes caused the patient's head to be turned to each side during the exposure. The success of the examination is explained mathematically in his paper and it is shown that to obtain the best results the head should not be turned more than $10^{\circ}$ to each side 


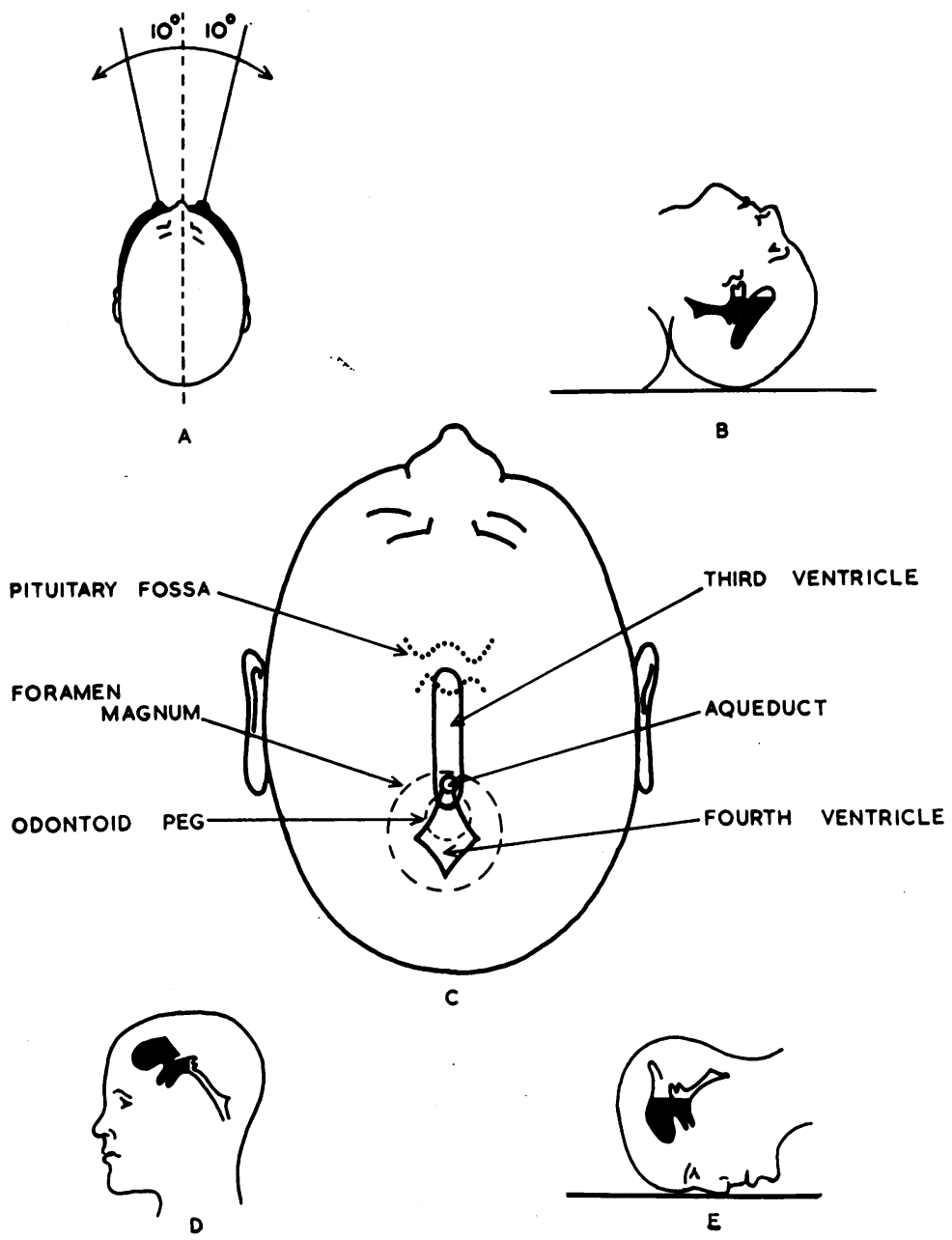

FIG. 9.-Autotomography: (A) Demonstrates the rotational movement of $10^{\circ}$ to each side of the midline, which is necessary to produce the tomographic effect. (B) Autotomography in the hanging head lateral position to demonstrate the anterior end of the third ventricle. (C) $\mathrm{A}$ diagram of the median ventricles illustrates how structures in the midline will move less than those off the midline during rotation. In addition the relationship of the median ventricles to the fulcrum of rotation in the plane of the odontoid process is shown. (D) Shows the patient in the erect lateral position during encephalography. The aqueduct, fourth ventricle and posterior part of the third ventricle are outlined. (E) During ventriculography the patient in the prone position demonstrating the same structures as in (D).

during the exposure (Figs. 9 and 10). No other technical details are given and only the examination of the fourth ventricle was carried out. Since 1956 this manœuvre has been used at the National Hospital for Nervous Diseases, London, and the technique was elaborated and perfected in conjunction with Miss Hamilton, Superintendent Radiographer at that hospital.

The technique as described by Ziedses des Palantes was first brought to the author's notice by Dr. Morris Simon (1956). The mathematical explanation given by Ziedses des Palantes in his paper can be simplified. The fulcrum of rotational movements of the skull lies on a line drawn upwards through the long axis of the odontoid peg; the median ventricles are not far removed from this line in an anterior or posterior direction (Figs. 9 and 10). Thus rotational movement of the head causes the structures near the fulcrum of rotation to move less than those structures far removed from it. In addition, the median ventricles move far less than structures in the midline when rotational movement occurs. The movement therefore blurs overlying structures, e.g. the mastoid bones, air-filled temporal horns or air-filled cortical sulci. The observations on this manœuvre show that the best movement of the head to obtain the effect is exactly that made when the head is shaken as if saying 'no'. The movement should be smooth, should not exceed $10^{\circ}$ to each side and should take a little over one second to complete. It should be repeated four or five times smoothly and continuously just before the beginning of the radiographic exposure, finishing well after the completion of the exposure. Co-operative patients are able to perform this movement quite well, hence the term autotomography. Schuarz facilitates this movement by attaching reins to the encephalographic halter used with the Lysholm Skull Unit and assists the 


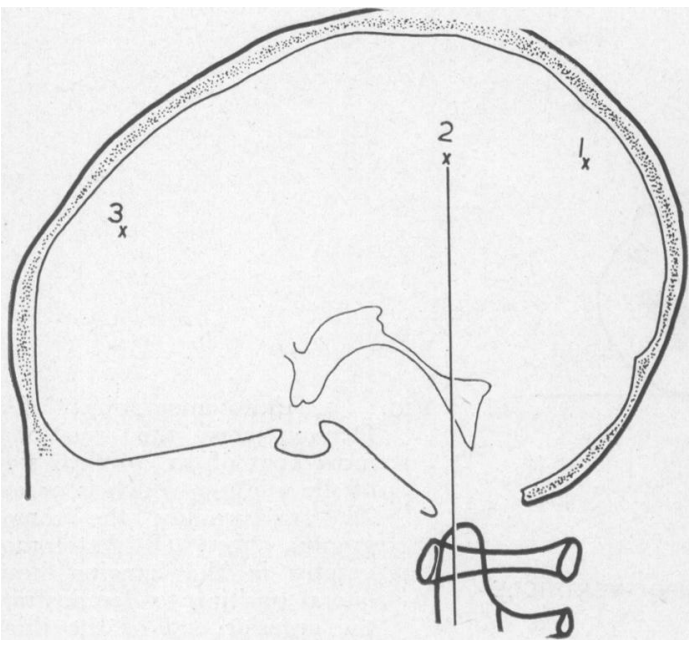

FIG. 10.-This diagram shows the relationship of the odontoid peg to the median ventricles. The points $I$ and 3 will move much more than point 2 when the head is rotated. As the median ventricles are not far removed from the point 2 they will move less than structures in front or behind, and the tomographic effect is enhanced.

rotation of the patient's head by this means (Schuarz, 1959).

All the examinations have been carried out on a Lysholm Skull Unit and the factors which give the best results are the following: $5 \mathrm{kV}$. are added to the figure needed for the usual lateral skull exposure; the number of milliamps per second is kept the same, but the exposure time is increased from $I \frac{1}{2}$ to $3 \mathrm{sec}$. The manouvre can be carried out in the supine, hyperextended supine and prone lateral positions. Using the horizontal ray lateral projection, the anterior end of the third ventricle, the region of the foramen of Munro, the posterior end of the third ventricle, the aqueduct and fourth ventricle can be examined (Figs. I1, 12, 13 and 14). In addition, as in the case of the median ventricles, the median subarachnoid structures are demonstrated more clearly than with a simple lateral radiograph. More recently, other authors have given accounts of this procedure (Jing and Schechter, I960; Mahoney, 1960).

\section{Infantile Hydrocephalus}

The introduction of the Spitz-Holter valve operation, which appears to arrest infantile hydrocephalus in $70-90 \%$ of cases (Spitz, 1959; Macnab, 1959), has stimulated interest in this condition and emphasized the need for accurate and early diagnosis. In a recent paper (Lawrence, 1960) it is stated that hydrocephalus is more often acquired than congenital.
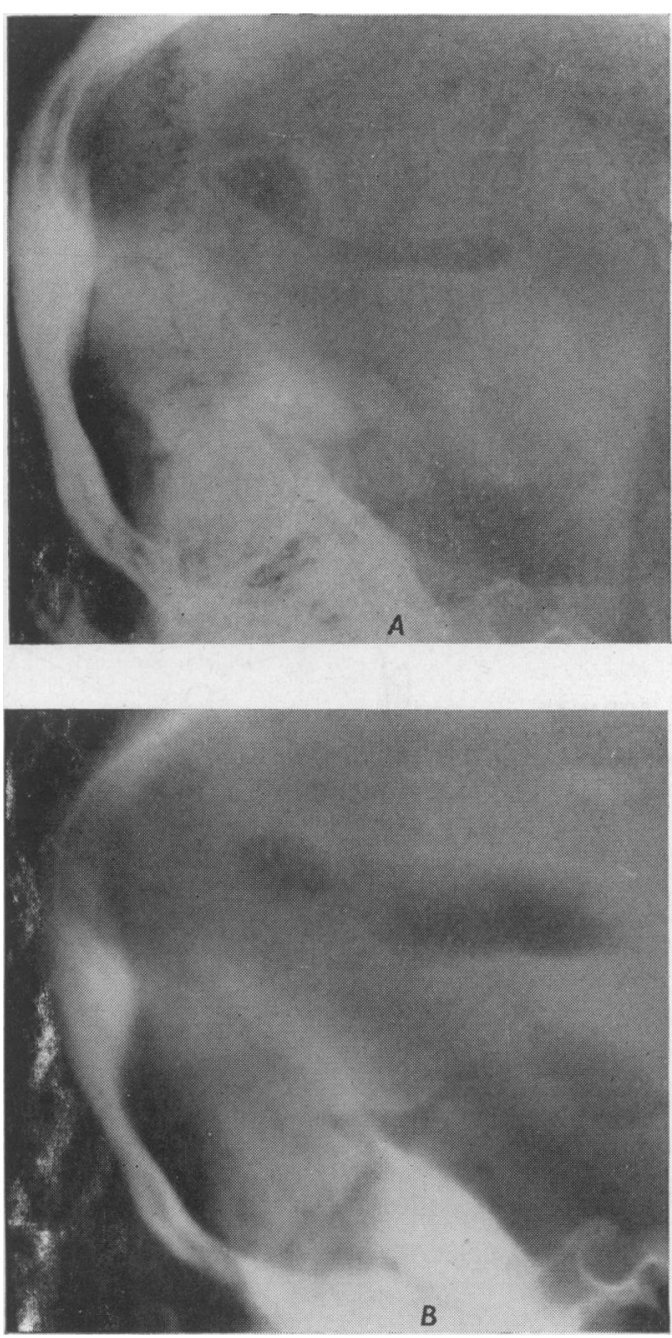

FIC. I1.-(A) Filling erect lateral radiograph during encephalography. Posterior fossa median ventricles not well seen. (B) Autotomogram in same position. These ventricles are much more clearly demonstrated.

Spontaneous arrest of hydrocephalus has been reported by many authors. Bucy (1950) quotes $10 \%$ as the incidence of spontaneous arrest and other authors indicate a $30 \%$ remission rate (Forrest, Laurence and Macnab, 1957). In the sc:ies quoted by Lawrence (1960), seen over a pciiod of 20 years by Mr. Wyllie McKissock, 8I out of 182 patients were alive and the hydrocephalic process had undergone spontaneous arrest. A high proportion of these had normal intelligence.

Lawrence also states that obstruction in the basal cisterns is the most common cause of hydroccphalus and is usually due to a congenital mal- 

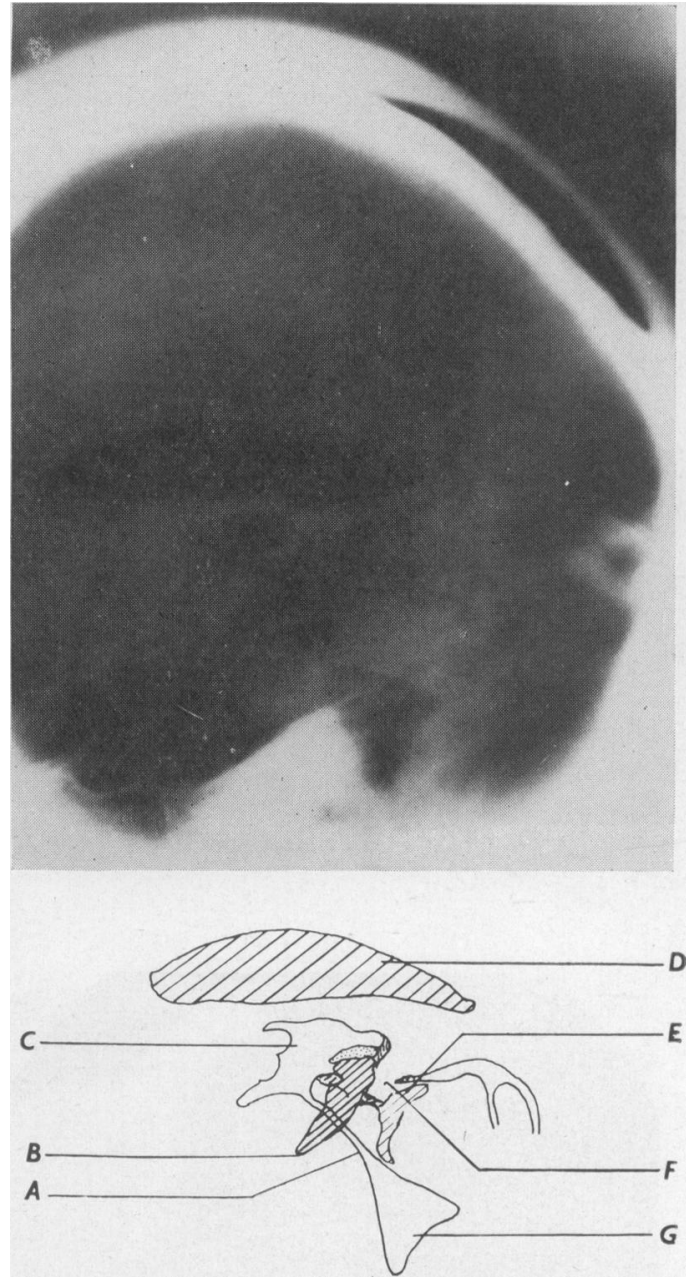

FIG. 12.-Autotomography: Filling erect lateral radiograph during encephalography. The aqueduct (A) fourth ventricle $(G)$ and third ventricle $(C)$ are well seen. A blurred shadow crosses the aqueduct, this is in the wing of the ambient cistern (B). The quadrigeminal plate cistern contains air (E). The quadrigeminal bodies are outlined and a filling defect in the posterior part of this cistern is caused by the vein of Galen $(F)$. The lateral ventricles (D) also contain air.

formation or is inflammatory in origin. The Arnold Chiari malformation is associated with a spina bifida cystica and the association with congenital aqueduct stenosis is also noted. It is pointed out that the commonest single cause of post-inflammatory hydrocephalus is subarachoid bleeding resulting from birth trauma, anoxia or head injury. Frequently, however, in the absence of such a history, hæmosiderin deposits are found in the basal meninges at post-mortem, suggesting that the cause of the hydrocephalus was traumatic in origin. The narrow aqueduct is susceptible to the presence of bleeding or pus and may become stenosed as a result of the presence of these substances within its narrow lumen. If only this part of the ventricular system is permanently damaged it is difficult to differentiate an acquired from a congenital stenosis except at autopsy.

Obstruction in the region of the tentorial hiatus can produce dilatation of the interpeduncular and chiasmatic cisterns which may cause a marked filling defect in the floor of the third ventricle, particularly in the infundibular recess. This appearance must not be mistaken for a tumour or arachnoiditis - the pathogenesis of this cisternal dilatation is difficult to explain (Fig. 15).

In an account of choroid plexus papillomas in childhood (Crofton and Matson, 1960), it is stated that these benign tumours produce excessive amounts of cerebro-spinal fluid and almost always produce hydrocephalus. They are frequently present in the lateral ventricles and unless these large ventricles are adequately filled with air it is easy to miss the filling defect caused by the tumour. The cerebro-spinal fluid is often xanthochromic. One case in this series occurred in a cyst communicating with, but outside, the lateral ventricle. Good post-operative results were obtained and the majority of cases returned to normal after operation.

In view of the fact that it is important not to miss the small number of benign tumours which may occur in any series of hydrocephalics it is the present writer's opinion that a full neuroradiological assessment should be carried out. If the fourth ventricle and aqueduct have not been outlined by ventriculography, lumbar encephalography should be performed to demonstrate these structures and the subarachnoid cisterns.

The efficiency of the Spitz-Holter valve may mask benign conditions for a considerable period and make their correction more difficult if and when they are discovered. It would seem that it is wrong, therefore, to perform the Spitz-Holter operation unless an accurate diagnosis has been made and benign tumours excluded.

\section{Cerebral Angiography}

\section{Catheterization}

Elfin reviews the literature on this subject (Elfin, I960) and describes a technique of selective catheterization of the external or internal carotid via the common carotid by using a curved catheter. If the curve is directed posterolaterally the internal carotid artery is entered, and if the curve is directed anteromedially the external carotid. $\mathrm{He}$ reports an incidence of $1.2 \%$ with spasm but does not indicate how far the catheter is placed in the artery. Personal experience of carotid catheteriza- 


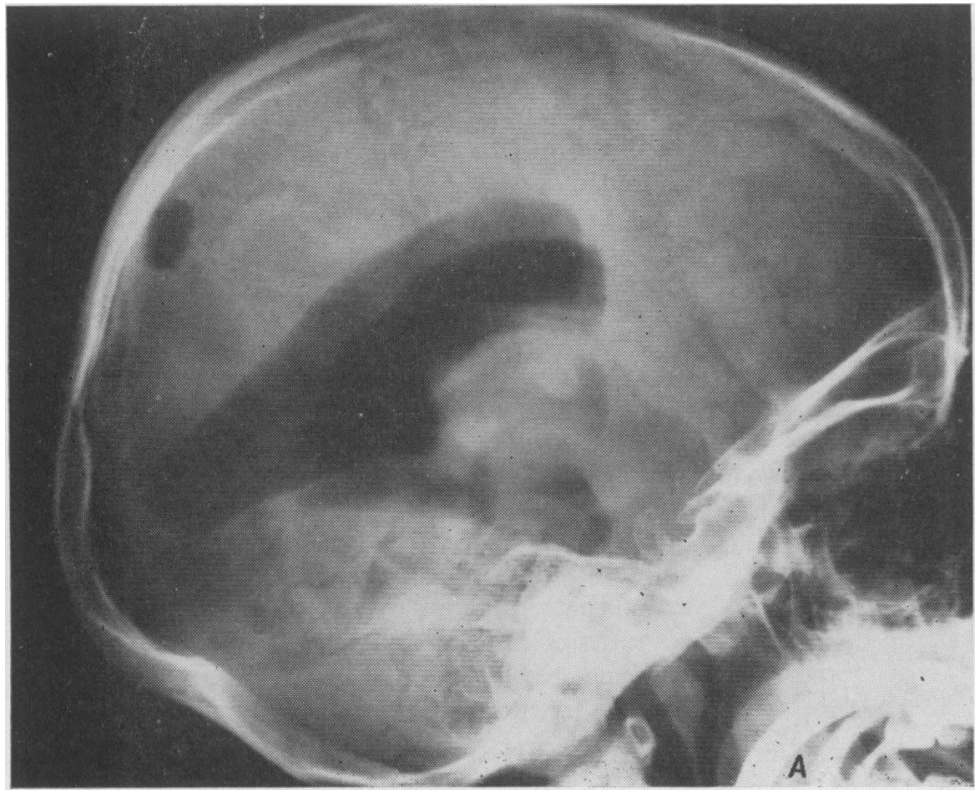

Fig. 13.-(A) Lateral prone radiograph of a ventriculogram in a child with signs of a posterior fossa tumour. The fourth ventricle is not well seen. (B) An autotomogram in the same position. The presence of a fourth ventricle tumour is clearly shown. This proved to be a medulloblastoma.

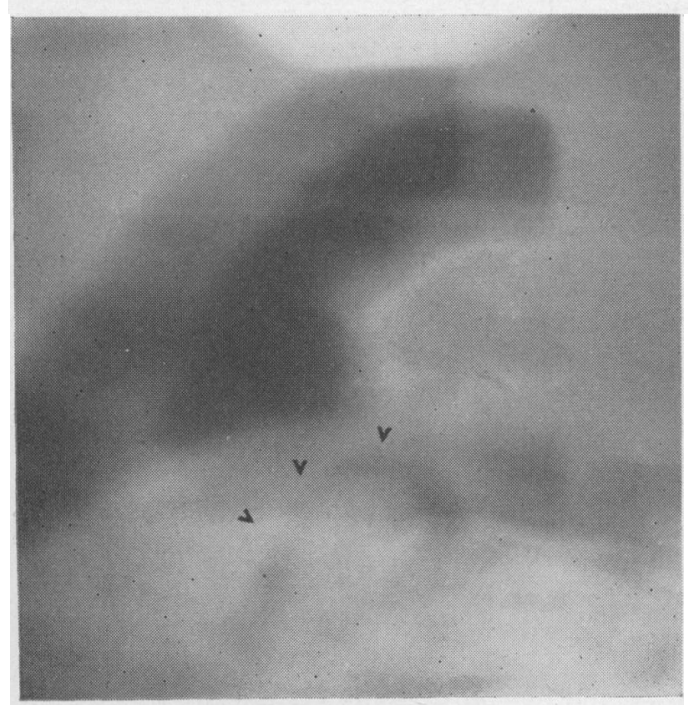

B

tion in an attempt to produce selective filling of the ophthalmic artery was as follows: A catheter with a curved tip was passed as far as was possible up the internal carotid artery. In all cases, marked spasm occurred at about the level of the first cervical vertebra, so much so that the blood flow v. as temporarily stopped until the catheter was withdrawn. One patient developed a transient hemiplegia as a result of this. Filling of the ophthalmic artery was no better than that obtained

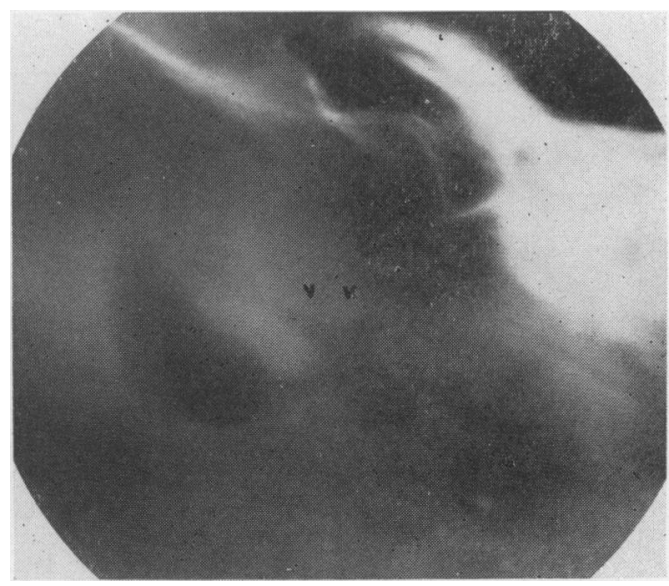

FIG. 14.-An autotomogram of an encephalogram in the lateral supine hanging head position to show the anterior end of the third ventricle. It is seen displaced backwards and upwards. The sella shows signs of a supra-pituitary lesion. 'This was found to be a cranio-pharyngioma.

with a simple percutaneous internal carotid artery puncture, and the technique was abandoned for this purpose. It is suggested that the nerve supply to the carotid artery is not uniform, as its various parts do not go into spasm with the same facility. Similar local sensitivity is seen in the 


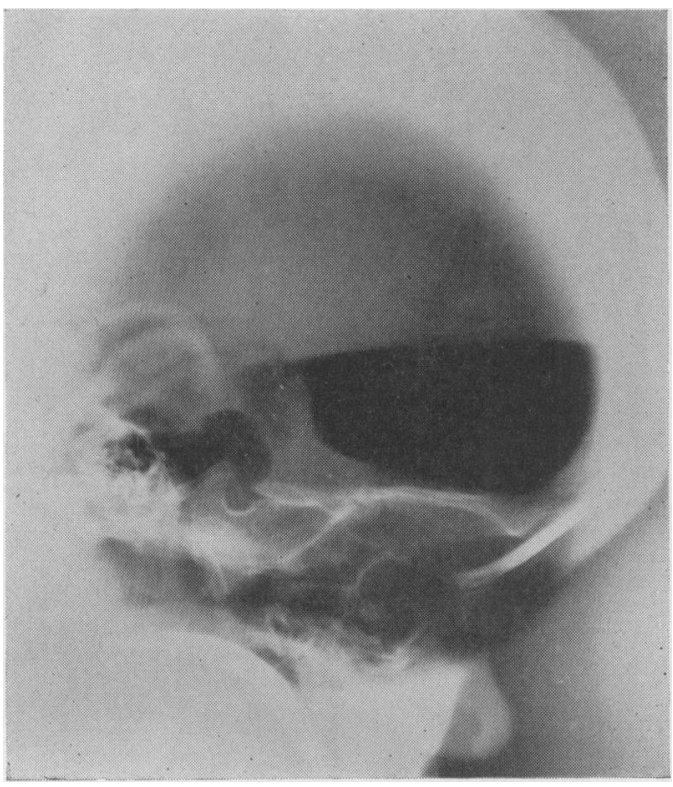

FIG. 1 5.-Hydrocephalus with gross dilatation of pontine, interpeduncular and chiasmatic cisterns obliterating the infundibular recess and displacing the third ventricle upwards. The region was explored and only dilated cisterns found with normal arachnoid and pia mater. This must not be called arachnoiditis-it is seen frequently in hydrocephalus where there is no obstruction below the tentorial hiatus. Its pathogenesis is obscure.

spasm associated with subarachnoid hæmorrhage. This is often localized in the region of the bifurcation of the internal carotid artery.

\section{Percutaneous Puncture of the External Carotid}

In this technique (Ruggiero and Jay, 1958) the neck is rotated away from the side to be punctured and the cornu of the hyoid bone identified; the puncture is made on a line drawn from this point to the angle of the jaw with the head still in the rotated and extended position. Personal experience with this technique indicates that it is easy, and a high success rate is possible. It is of value in meningiomas and arteriovenous malformations.

\section{Filling of the Posterior Cerebral Artery}

Saltzman, in a series of papers on circulation through the anterior and posterior communicating arteries (Saltzman, 1959a, b, c), describes a technique for compression of the vertebral artery. ' One finger is introduced immediately above the clavicle behind the common carotid artery and is directed inwards; when the finger has covered about $2 \mathrm{~cm}$. of the transverse process it is firmly pressed still in the same position against the process'. In the small number of cases recorded when this manœuvre was performed during carotid arteriography, a number showed filling of the posterior communicating and posterior cerebral arteries. Blood flow in the opposite direction during vertebral arteriography, with carotid-artery compression resulting in filling of the posterior communicating artery and carotid siphon, has also been recorded (Krayenbuhl and Yasargil, 1957). Further observations by Saltzman show that the highest incidence of filling the posterior cerebral artery is achieved if:

I. The internal carotid artery is punctured.

2. Rapid serial angiography is performed.

3. Rapid injection of contrast medium through short needles is made.

4. Puncture complications are avoided.

5. Compression to the vertebral artery is applied.

In pathological states, contrary to other observations, the incidence of filling of the posterior cerebral artery was not lower in the presence of space-occupying lesions apart from those in the middle fossa. The percentage of instances in which this artery was filled was lower in the presence of aneurysms; spasm was suggested as the cause of this. The remarkable exception occurred in aneurysms on the posterior communicating artery where the percentage filling of this artery and the posterior cerebral artery was higher than normal; the explanation given is that the congenital defect of the muscular coat associated with the aneurysm formation may allow the passage of contrast medium (Saltzman, 1959). If there is an occlusion of an artery beyond the origin of the posterior communicating artery, then the percentage filling of this artery and the posterior cerebral artery is again more commonly observed. These papers indicate the dynamic state of the Circle of Willis.

\section{Sinography}

A simple technique for cerebral venous sinography by means of carotid arteriography using the hand changer on the Lysholm Skull Unit is given by Morris (1960). One hundred cases are described and the anatomy discussed. Oblique half-axial views are used and exposures made at about 6 sec. after the injection. Six abnormal cases are included, trauma, meningioma and middle ear disease being the ætiological agents. Using this technique, Morris (196r) had diagnosed cerebral thrombophlebitis and has had one case confirmed by autopsy. A disordered venous pattern was demonstrated with meagre filling of the main cerebral venous sinuses (Fig. 16). 


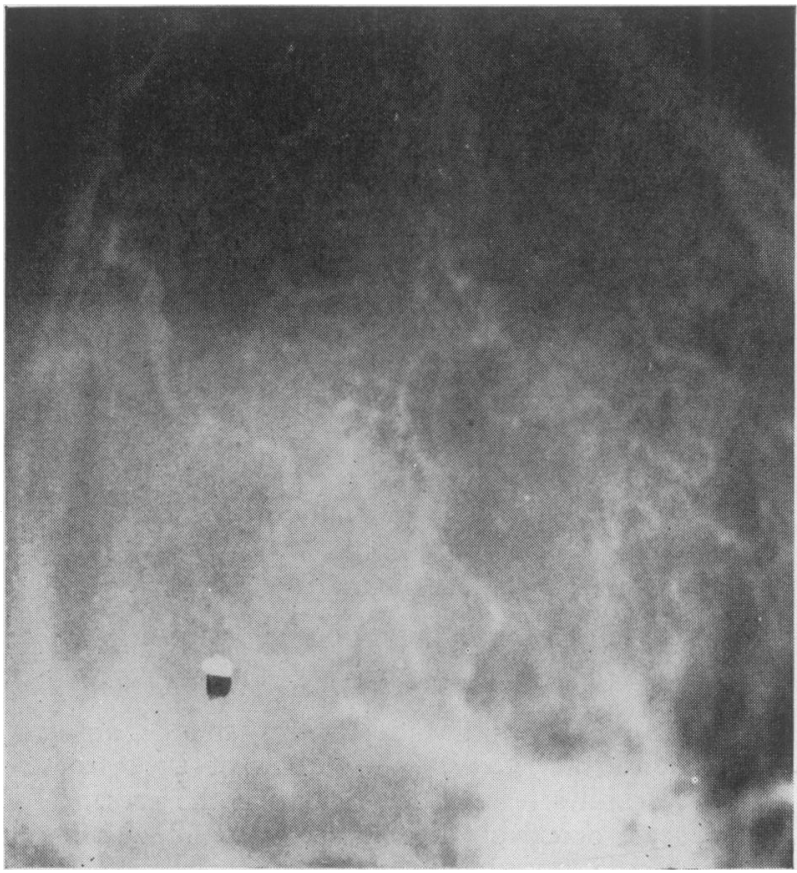

FIG. 16.-This illustration shows the late venous phase of a carotid arteriogram. A disordered pattern is seen, with poor filling of the venous sinuses. A superficial cerebral thrombophlebitis was found at autopsy.

\section{Rapid Serial Angiography}

The author has been impressed with the routine use of the Elema Schonander serial changer (AOT 24/30) for cerebral angiography in Scandinavia. Its value in the stroke syndrome and occlusive vascular disease is discussed later, and, in the monograph on this subject by Greitz (1956), its value in demonstrating minor pathological circulations was established. These may exist before vascular displacements occur. Wickbom (196I) studies the stroke syndrome using rapid serial angiography with profit. If minor strokes and primary intracerebral hæmorrhage were examined by this method, diagnostic accuracy would be increased, and in time there would be less acceptance that three lateral and two anteroposterior films are adequate in routine cerebral angiography. The quality of the films produced by this method in Sweden appeared to be as good as those produced by the hand changer. Particular attention is paid to the lenticulo-striate arteries (Andersen, 1958) and the deep cerebral veins.

\section{Orbital Phlebography}

The value of carotid angiography in orbital conditions has been described (Krayenbuhl, 1958). In this paper the normal radiological anatomy is shown and the appearances of space-occupying lesions and vascular anomalies depicted. This examination does not always give the maximum amount of information and recently a paper on orbital phlebography has shown that angiomas, particularly the cavernous and capillary type, may not show on carotid arteriography but do so (particularly when accompanied by a large varix) by phlebography of the angular vein. The technique depends upon the use of a fine needle having a short bevel attached to fine polythene tubing. Percutaneous puncture under local anæsthesia is made (Hobbs, Du Boulay and Davis, 1960). The length of the polythene tubing allows freedom of movement, thus compression of alternative pathways of venous drainage and continuous slow perfusion of saline is possible (Du Boulay, 196r).

\section{Congenital Anomalies of the Circle of Willis}

Anomalies of the Circle of Willis have been shown to occur in about $48 \%$ of all autopsies (Alpers, Berry and Paddison, 1959).

Sutton (1950) first described the angiographic appearances of the primitive trigeminal artery. More recently, Saltzman (1959) has classified the types. In some cases only the posterior cerebral arteries are supplied by the primitive artery, in others the whole of the posterior fossa blood supply may come from this primitive artery and the vertebral arteries may be absent or small. Morris (1959) has shown an absent vertebral artery in such a case.

Failure of development of the anterior branch of the primitive internal carotid artery which gives rise to the anterior and middle cerebral arteries is 

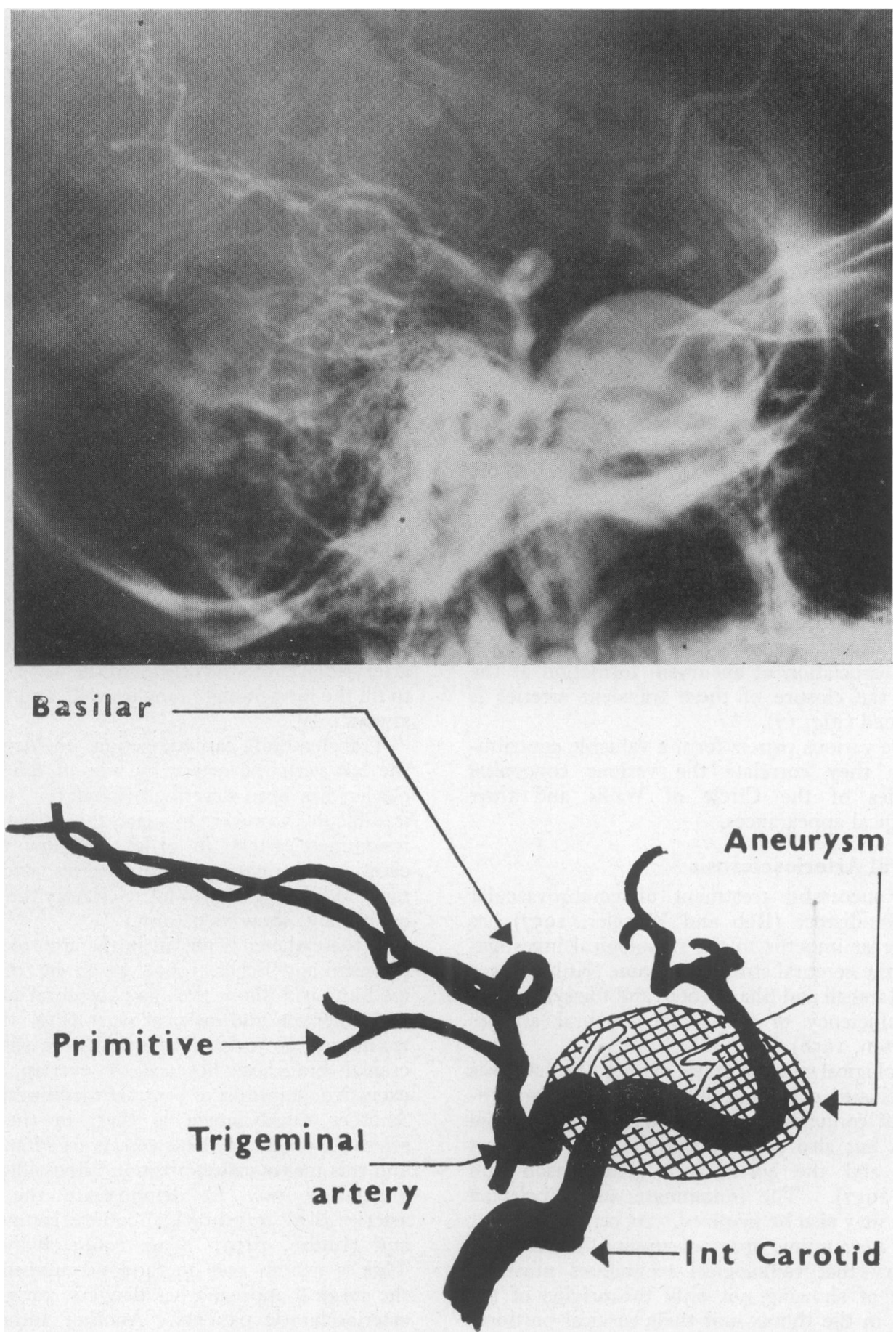

Fig. 17.-This radiograph illustrates the association of aneurysms with persistent primitive arteries. There is a large primitive trigeminal artery supplying the posterior fossa vessels with the exception of the inferior cerebellar arteries. A large subclinoid aneurysm is present and there is only poor filling of the branches of the internal carotid which peters out in the region of the anterior clinoid processes. 
associated with the presence of a primitive persistent olfactory artery and anomalies of the posterior cerebral artery (Berk, I96I). Two cases are described and the embryology succinctly discussed. The anterior parts of the anterior and middle cerebral arteries were missing. The territory usually supplied by these came in part from the primitive persistent olfactory artery (which arises in the region of the anterior communicating artery and courses supero-laterally) and via anastomoses from the posterior branches of the posterior cerebral artery. In addition, one of the cases had a plethora of small vessels in the region of the anterior communicating artery. It was suggested that these were anastomotic vessels.

A case with absence of the proximal part of one anterior cerebral artery is discussed-the ætiology suggested is $(a)$ persistence of the median artery of the corpus callosum, or $(b)$ simple atrophy of the proximal portion of the anterior cerebral artery.

Another angiogram is shown with three arteries arising from the region of the anterior communicating artery and it is suggested that one of these arteries is a persistent median artery of the corpus callosum, the other two being the right and left anterior cerebral arteries.

The association of aneurysm formation at the site of the closure of these transient arteries is mentioned (Fig. 17).

These various papers form a valuable contribution as they correlate the various congenital anomalies of the Circle of Willis and their radiological appearances.

\section{Cerebral Arteriosclerosis}

The successful treatment of cerebrovascular occlusive disease (Rob and Wheeler, 1957) has given great impetus to the radiological investigation of the cerebral stroke syndrome (Kuhn, I959; Bull, Marshall and Shaw, I960) and the syndromes of insufficiency of the major cerebral arteries (Elkington, 1958).

Pathological studies have shown that stenosis and occlusion occur not only at the main branchings and conjugations of the four major cerebral arteries but also at their origins from the great vessels and the aortic arch (Hutchinson and Yates, 1957). The innominate and subclavian arteries may also be involved. As cerebrovascular disease may mimic space-occupying lesions, it is necessary that radiological techniques must be capable of showing not only the origins of the arteries in the thorax and their cervical portions, but also, in detail, the intracranial branches. The numerous techniques which have been described indicate the inability of any one of them completely to achieve this end.

It is a good principle not to puncture the main cerebral arteries if they are considered to be the site of occlusive arteriosclerosis, as trauma to them might place in jeopardy a brain whose blood supply may be precarious. In the light of this, Baker ( 1960 ) describes a method of performing complete cerebral arteriography. Percutaneous puncture of the right subclavian or innominate artery will demonstrate the right carotid and right vertebral artery; this may be followed by a left common carotid artery puncture and a left subclavian artery puncture (to show the left vertebral artery). Thus only one of the four cervical cerebral arteries will be directly punctured. Three of the four, however, will be shown in their total length, the exception being those parts of the left common carotid artery below the site of the puncture. Percutaneous puncture of the subclavian arteries to show the vertebral arteries has also been described by others (Barbieri, I959; Morris, 1960). The major disadvantages associated with percutaneous subclavian puncture to demonstrate the vertebral arteries include the risk of production of a pneumothorax (this has a low incidence in expert hands) and the inconstancy with which the intracranial branches are adequately demonstrated. It is necessary to place the needle in the subclavian artery adjacent to the ostium of the vertebral artery to fill the intracranial branches well, and this is not always easy.

Transfemoral catheterization to demonstrate the left vertebral artery by way of the left subclavian has been described (Lindgren, 1956). It is difficult, however, to place the catheter in the innominate artery in order to show the right carotid and right vertebral arteries, and by this method it is not easy to fill selectively the common carotid and show its ostium.

If the catheter is placed in the arch of the aorta (Gensini and Ecker, 1960), 25-30 c.c. of contrast medium will show the main cerebral arteries in their thoracic and cervical parts (Fig. I 8). This technique, however, is unsuitable to show intracranial branches, because of overlap, and the excessive quantities of contrast medium necessary. Another disadvantage is that, in the arteriosclerotic subject, the iliac vessels are often tortuous and this makes catheterization impossible.

Another way to demonstrate the cerebral arteries is by transbrachial catheterization (Pygott and Hutton, 1959; Begg, 1960; Kuhn, 1960). This is not an easy method percutaneously and the surgical approach has dangers, particularly in arteriosclerotic patients. Another author (Berk, ig60) recommends retrograde catheterization of the common carotid artery and, by this means, demonstrates the vertebral artery. Catheterization of the carotid artery inevitably results in more trauma to the artery than a simple puncture. In 

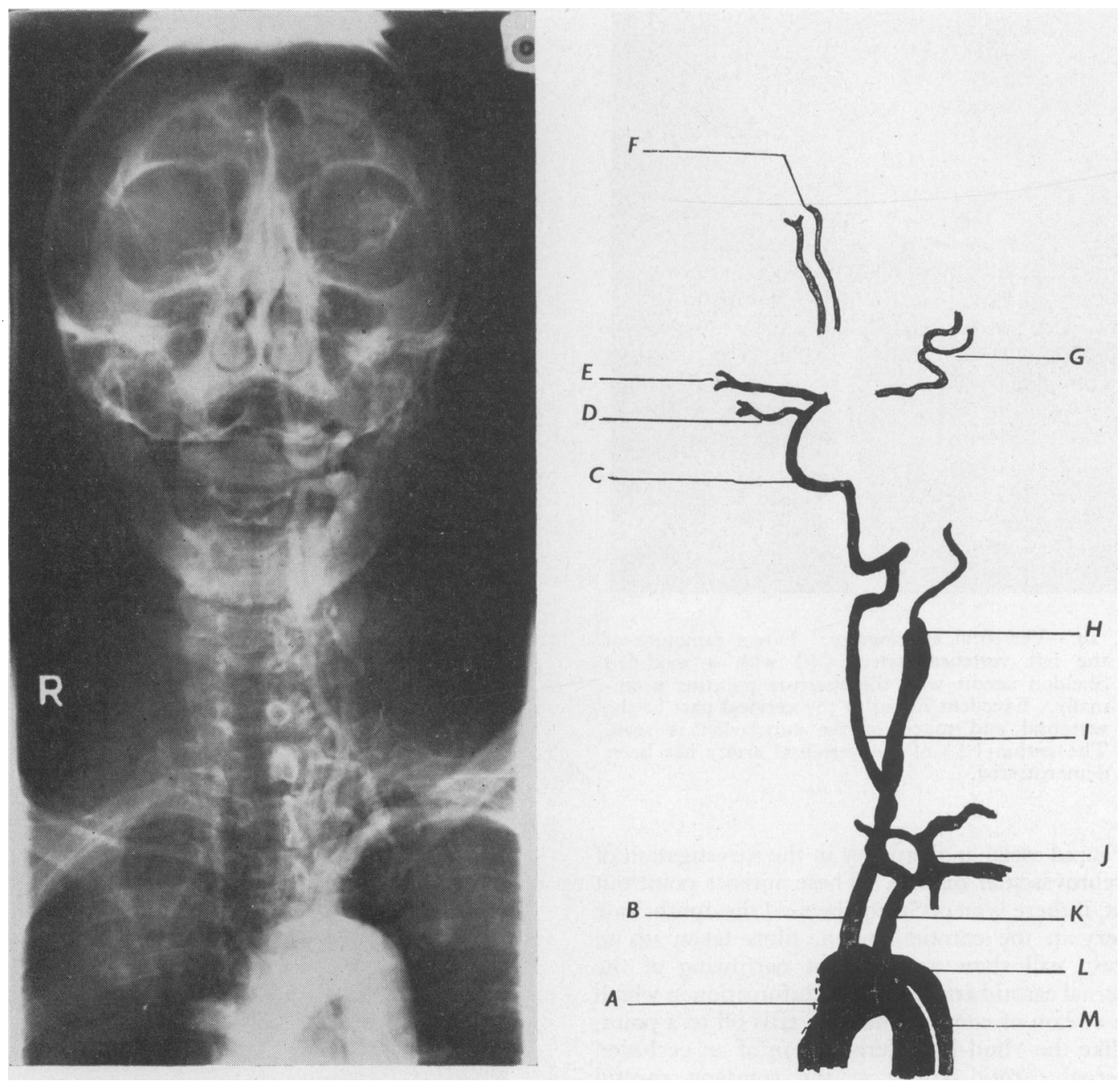

Fig. I 8.- Transfemoral aortogram to demonstrate the cerebral vessels. A 56 -year-old male had a short history of a fluctuating left hemiplegia which rapidly became complete and the patient comatose. Then the right radial and subclavian pulses became impalpable. It was decided that direct cervical cerebral artery puncture would be dangerous and accordingly a transfemoral aortogram was carried out. (A) Shows irregularity of the aortic arch in the region of the innominate artery which has not filled; (B) shows the left common carotid artery; (C) represents the basilar artery; (D) the superior cerebellar; (E) the right middle cerebral; (F) the anterior cerebral arteries; (G) the left middle cerebral; $(\mathrm{H})$ the stenosed left internal carotid artery-a branch of the left external carotid artery overlies this stenosis; (I) the vertebral artery; (J) the thyro-cervical trunk; (K) the left subclavian artery; (L) the aortic knuckle; (M) the position of the catheter in the aortic arch. These appearances were confirmed at operation.

the radiological investigation of cerebral arteriosclerosis, therefore, this is not considered a good method.

The direct puncture of the four main cerebral arteries, although it has theoretical disadvantages, is a practical technique. Refinements of this technique have made vertebral arteriography comparable with carotid arteriography (Sheldon, 1956; Swann, 1958). Furthermore, retrograde injection of the contrast medium by turning the modified Sheldon needle through $180^{\circ}$ will show with ease the ostia of the vertebral arteries and, if this needle is used in carotid arteriography, the ostia of the carotid arteries can also be shown (Swann-in press) (Figs. I9 and 20).

Two recent papers on the misinterpretation of the site of carotid stenosis (Murphy and Shillito, I959; Newton and Couch, 1960) indicate a place 


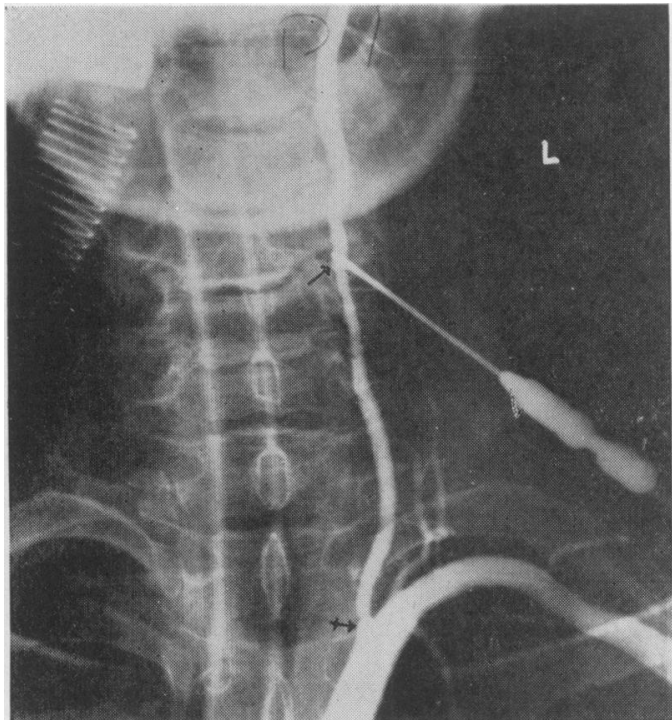

FIG. 19.- Vertebral arteriogram. Direct puncture of the left vertebral artery $(\uparrow)$ with a modified Sheldon needle with the aperture pointing proximally. Excellent filling of the cervical part of the vertebral and much of the subclavian is seen. The ostium $(\uparrow)$ of the vertebral artery has been demonstrated.

for rapid serial angiography in the investigation of cerebrovascular disease. These authors point out that, if there is an occlusion beyond the ophthalmic artery in the carotid siphon, films taken up to 4 sec. will show an apparent narrowing of the internal carotid artery near the bifurcation in which the stream of contrast medium tails off to a point, unlike the 'bud-like' termination of an occluded internal carotid artery at the common carotid bifurcation. If, however, films are taken up to 8 sec., the real site of occlusion will be shown in the region of the carotid siphon.

The use of cerebral angiography in the diagnosis of acute cerebral catastrophes (cerebral stroke) has resulted in many papers. In a series of 80 cases (Bull et al., 1960) the great value of angiography has been demonstrated - a considerable percentage of the cases were radiologically abnormal, and in these cases angiography showed the inaccuracy of clinical diagnosis. There was a low incidence of serious complications with direct puncture. It has been stated that one in four strokes is due to a segmental atheromatous occlusion of the cervical carotid (Kuhn, 1959). The author of this paper also states, 'It is my belief that cerebral angiography should be considered a vital and routine part of the medical management of patients developing hemiplegia or other manifestations of
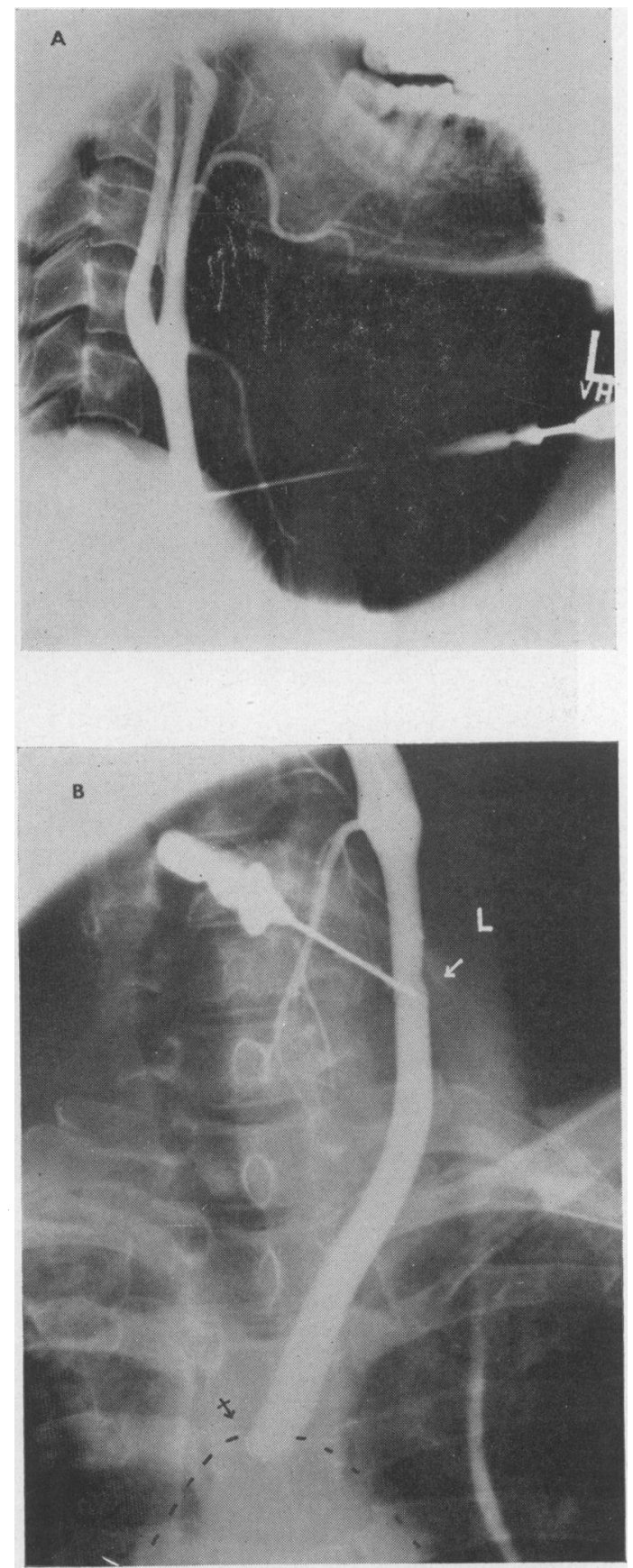

FIG. 20.-Common carotid arteriogram, using the modifield Sheldon needle: (A) The aperture of the needle pointing distally and demonstrating the bifurcation of the left common carotid artery. (B) The aperture of the needle directed proximally $(\uparrow)$ and demonstrating the ostium $(\uparrow)$ of the left common carotid artery. 
stroke with or without intracranial bleeding'. Another statement made in this paper with regard to cerebral hæmorrhage is, "It is not by accident that early diagnosis by angiography, coupled with an aggressive surgical approach, has lowered mortality rates from almost $100 \%$ to about $50 \%$ in elderly exceedingly ill patients with massive brain hæmorrhages'. A series of 244 capses of intracerebral hæmorrhage of which 164 were operated on (McKissock, Richardson and Walsh, 1959) gave interesting results. Only a $70 \%$ success rate was achieved in localizing the hæmorrhages by angiography. Of the 53 negative angiograms 22 had basal ganglia disruptive hæmorrhages and 33 had significant hæmatomas which were removed at operation. It is stated in this paper, "That angiography was too unprecise and a diagnostic method other than surgical intervention should be used ', but Wickbom (1961) uses rapid serial angiography routinely, varying the number of films with each case. In the cerebral stroke syndrome exhaustive studies are made with a larger number of films and minor arterial and venous abnormalities are often demonstrated in the smaller intracranial vessels.

Arteriosclerotic basilar stenosis and occlusion has been described (Elkington, 1958; De Bakey and Crawford, 1958). The radiological appearances may be quite characteristic with the formation of an abundant collateral circulation from the inferior cerebellar arteries to the posterior cerebral arteries. The anastomotic vessels have the typical tightly tortuous appearance of collateral vessels seen elsewhere in the body. These vessels are small, and films of good quality are necessary to demonstrate them. The present writer has seen four cases. The occlusion usually occurs at the conjugation of the vertebral arteries and often involves the whole length of the basilar artery. One of these cases has been confirmed at autopsy (Fig. 2I).

In summary, the radiological investigation of cerebrovascular arteriosclerosis may demonstrate among other things remedial arterial disease. The radiologist should work in close association with the clinician and, if clinical examination or ancillary tests indicate a precarious blood supply to the brain, it may be advisable not to attempt direct puncture of the major cerebral arteries, alternative arteriographic methods being available. Expert surgical and anæsthetic aid is needed if deterioration occurs after arteriography, in order that emergency thrombo-endarterectomy can be carried out. The angiographic investigation of the stroke syndrome and primary intracerebral hæmorrhage are considered important by many authorities, while rapid serial angiography may be valuable.
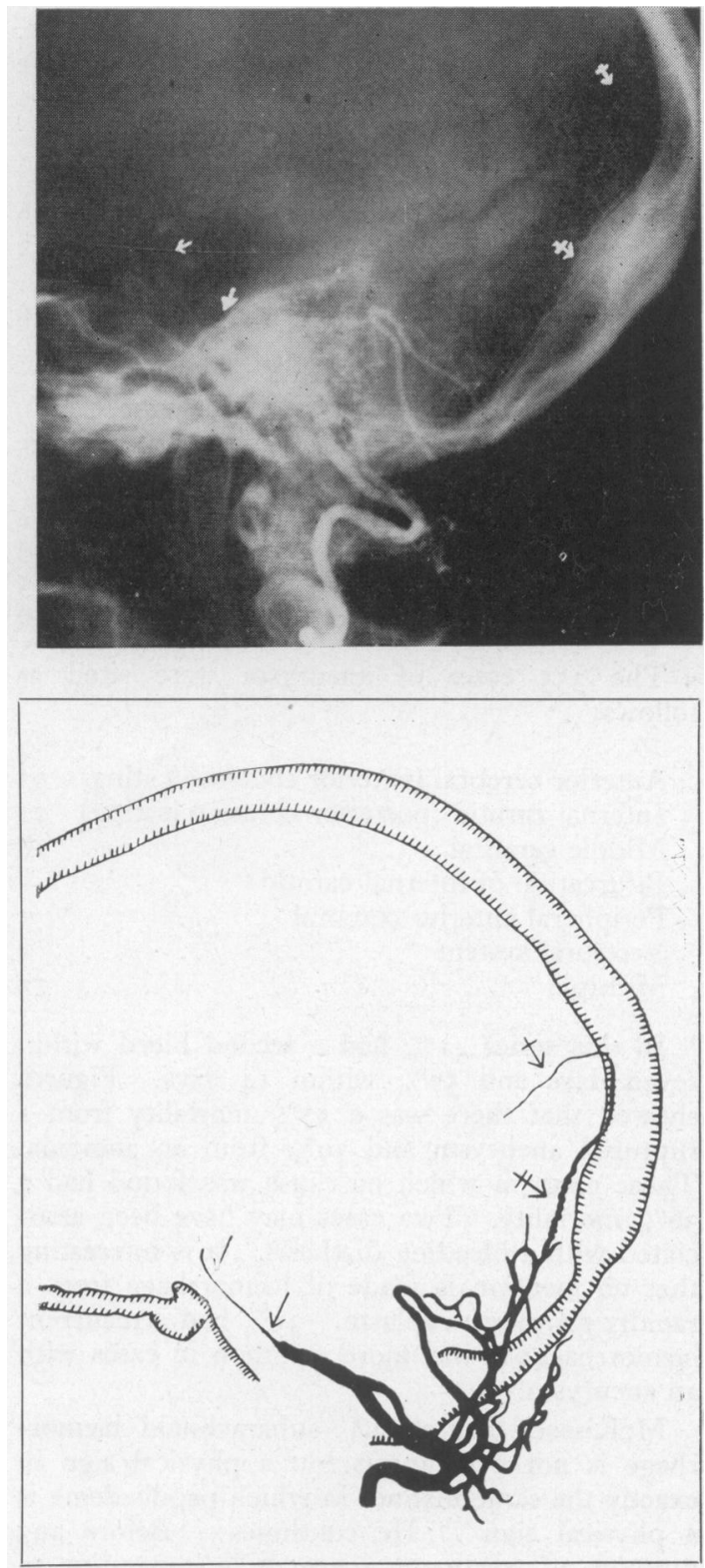

FIG. 21.-Vertebral arteriogram showing wire-like narrowing of the distal part of the basilar artery $(\uparrow)$. Tortuous collateral vessels are seen passing over the posterior $(\neq)$ and superior $(\uparrow)$ surfaces of the cerebellar hemispheres.

\section{Subarachnoid Hæmorrhage}

McKissock and his colleagues have given accounts of their findings in over 2,000 cases of subarachnoid hæmorrhage. These papers are 
probably the most authoritative in the literature. The causes of 2, 1 6 cases of this physical sign are given (McKissock et al., 1959).

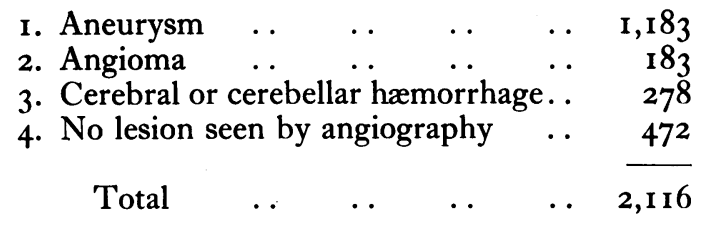

In another paper (McKissock and Paine, 1959), in a total of 781 cases of subarachnoid hæmorrhage the following percentages were given:

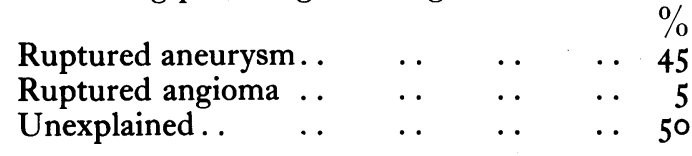

The 355 cases of aneurysm were sited as follows:

Anterior cerebral (anterior communicating) $\begin{aligned} & \% \\ & 3 \mathrm{I}\end{aligned}$

Internal carotid (posterior communicating) 2I

$\begin{array}{llllll}\text { Middle cerebral } & \ldots & \ldots & \ldots & \ldots & \text { I8 }\end{array}$

$\begin{array}{llll}\text { Bifurcation of internal carotid } & \ldots & \ldots & 8\end{array}$

Peripheral anterior cerebral $\quad \ldots \quad \ldots 2^{2}$

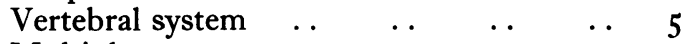

$\begin{array}{lllllll}\text { Multiple } & \ldots & \ldots & \ldots & \ldots & \ldots & \text { I } 5\end{array}$

In this series $41 \%$ had a second bleed within seven days and $57 \%$ within 14 days. Figures showed that there was a $57 \%$ mortality from a ruptured aneurysm and $39 \%$ from an angioma. Those cases in which no cause was found had a $36 \%$ mortality. Two cases may have been associated with a bleeding diathesis. It is interesting that no mention is made of hæmorrhage from a rapidly growing neoplasm. $35 \%$ had a recurrent hæmorrhage; it was more common in cases with an aneurysm.

McKissock has stated 'subarachnoid hæmorrhage is not a diagnosis but a physical sign in exactly the same manner in which papillodema is a physical sign'. He continues: 'Before any question of treatment arises, it is imperative to establish the pathological diagnosis'. Immediate bilateral carotid arteriography is preceded by a Matas Test (carotid compression). Vertebral arteriography under general anæsthesia is carried out 48 hours later if the carotid arteriograms are negative (McKissock in McKissock, Crawford, Bull and Perrett, 1959):

In the same symposium (Bull and Perrett, 1959) the accuracy of diagnosis by angiography in a series of 210 cases of intracranial arterial aneurysm was analysed. All these cases were examined at autopsy. In $89 \%$ the aneurysm was accurately localized by angiography. The remaining $11 \%$
were divided into five groups:

I. Spasm. There were five cases in this group in which spasm prevented filling of the aneurysm. This is a rather high figure but: these workers carry out immediate arterios? graphy after the diagnosis has been cone firmed by lumbar puncture and caroti compression tests performed.

2. Observer Error. In six cases the aneurysn was not observed.

3. Inadequate Examination. Four cases had an inadequate examination.

4. Normal Arteriogram. In another four cases the arteriograms were normal even i⿱ retrospect; at autopsy, however, the aneurysms discovered were small.

5. Multiple Aneurysms. Four cases had tw. aneurysms, only one of which was observed ${ }_{A}^{v}$

No aneurysm in this series had failed to fill at angiography by the presence of clot within it andw the observation is made that this must be accepted with reserve as a cause of non-filling of arf aneurysm.

The value of special projections is stressed ant compression of the opposite carotid artery is carried out during arteriography to establish theo patency of the anterior communicating artery. 응

The conclusion reached in this paper is that the potential accuracy in localizing aneurysms $96 \%$ if observer error is minimized and adequate projections of the cerebral vessels obtained.

\section{Myelography}

Myelography with Positive Contrast Media

It is still a common practice to remove ethy iodophenyl undecylate ('Myodil') after myeloz graphy, the lumbar puncture needle being left in position during the examination. It is not con $\frac{3}{3}$ sidered possible to perform a myelogram ade quately in such circumstances. Furthermore, it is thought unnecessary (Davies, I95I) and harmfub to remove the contrast medium because $(a)$ the subarachnoid space is subjected to additionab trauma, (b) Myodil pulmonary embolism max occur during its removal, $(c)$ if an examination is inadequate or equivocal the Myodil may be re screened many weeks after, and $(d)$.the arachnoidr reaction to the contrast medium is only of academic importance (Swann, I960).

Wiedenman and Fortschr (1960) have made the observation that jugular compression wiff cause Myodil to float against the force of gravity in the spinal fluid. Accordingly, this author has? devised a technique to demonstrate the upper- andio mid-thoracic regions. The contrast medium is tilted into the cervical lordosis, the patient is therif placed horizontally and the jugular veins are 


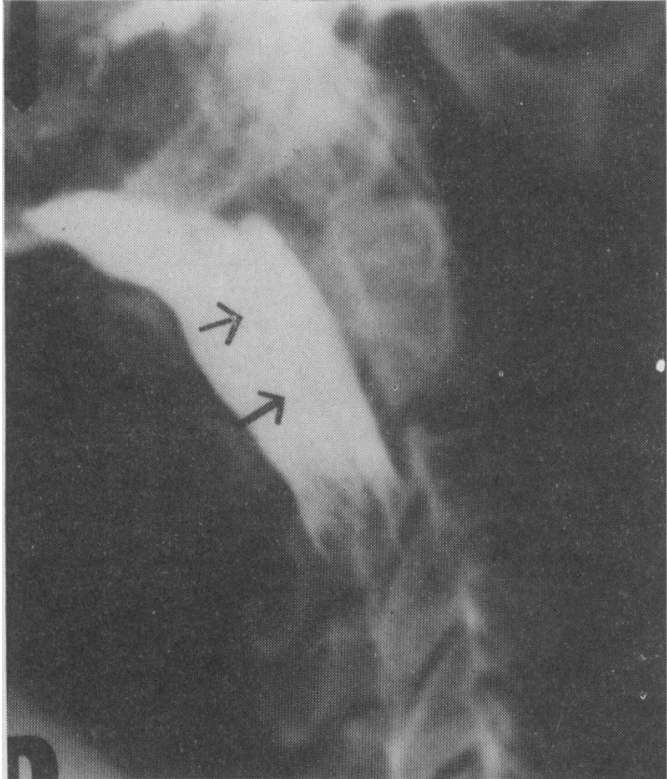

FIG. 22.-Cisternal myelogram. The dentate liganents are shown as linear translucencies $(\uparrow \uparrow)$ above a ccmplete block caused by an intramedullary cervical tumour.

compressed. The contrast medium will then rise towards the summit of the thoracic kyphosis and beyond it. This is a very useful manœuvre, particularly if used with lateral screening.

The dentate ligaments have been identified at myelography (Teng, 1960) and it is noted that they may be displaced and distorted by space-occupying lesions including disc protrusions. They give negative linear shadows which are best seen in the lateral view (Fig. 22).

In an account of the post-operative findings after lumbar-disc removal using water-soluble contrast media (Cronqvist, I959), the difficulties in distinguishing between post-operative scarring and recurrence of disc herniation are emphasized. (The use of water-soluble contrast media for myelography is considered inadvisable because it is necessary to produce spinal anæsthesia to perform this, examination.)

Arachnoiditis seldom causes a complete block, but a case has been described in which there was a large, solitary filling defect causing a complete block but without cord displacement (Mulvey, 1960). It is suggested that this combination might be a diagnostic feature of arachnoiditis. This suggestion is not accepted, as often with a complete block, cord displacement may not be revealed. Cystic arachnoiditis, however, was confirmed at laminectomy. It is unusual for arachnoiditis to be manifested by a single filling defect; characteristically there are multiple smooth-walled sacs which may fill and empty with tilting, and the irregularities in the Myodil column produced by adhesions will be present. It must be very rare for a single cyst to produce a defect without other cysts becoming contrast-filled, thus enabling a diagnosis to be made. Extensive radiological lesions are often present with a paucity of clinical signs. Scalloping of the posterior aspects of the bodies of the vertebræ may occur (Murray, 1959).

Frequently there is no history of a precipitating cause and in these cases only cyst formation may occur. These cysts may occur subdurally, extradurally and along the nerve root sheaths beyond the bony spine (Figs. 23 and 24). They are probably of a congenital nature and the suffix 'itis' is a cloak for ignorance. It is suggested that such cases may have a common ætiology with primary intracranial arachnoid cysts (Oliver, 1958). Unlike the intracranial cysts, however, underlying atrophy is not seen, and spinal arachnoid cysts may protrude through gaps in the dura.

Angiomas. Lombardi and Migliacavva (1959) record 18 cases of angiomas of the cord. They stress the necessity of using adequate amounts of Myodil (Fig. 25). Venous, arterio-venous and arterial types are described. The venous type is the most common. Thirteen cases were confirmed at operation; seven were venous, two were arterial and four were arterio-venous. Those in the cervical region are more often arterio-venous or arterial and may give rise to subarachnoid hæmorrhage. They can be demonstrated by vertebral arteriography (Morris, 1960).

\section{Myelography with Air}

This method of myelography is used extensively in Scandinavia and in some centres in France and Czechoslovakia. It is time-consuming and requires large numbers of films with tomography in the tilted position. Experience with this technique shows it to be excellent in demonstrating atrophy of the cord and small mid-dorsal posterior spaceoccupying lesions. It has been shown (Klefenberg and Saltzman, 1959) by air myelography that a pathognomonic appearance in syringomyelia may be demonstrated, the cord being narrow and atrophied below the widening produced by the syrinx. These appearances are not seen with other intramedullary tumours.

Jirout (I959a and b) has made a detailed study of the cervical cord and its movements as demonstrated by air myelography. He also has described a characteristic displacement of the cervical cord when there is axial dislocation of the brain stem along the clivus as the result of tumours. It must 


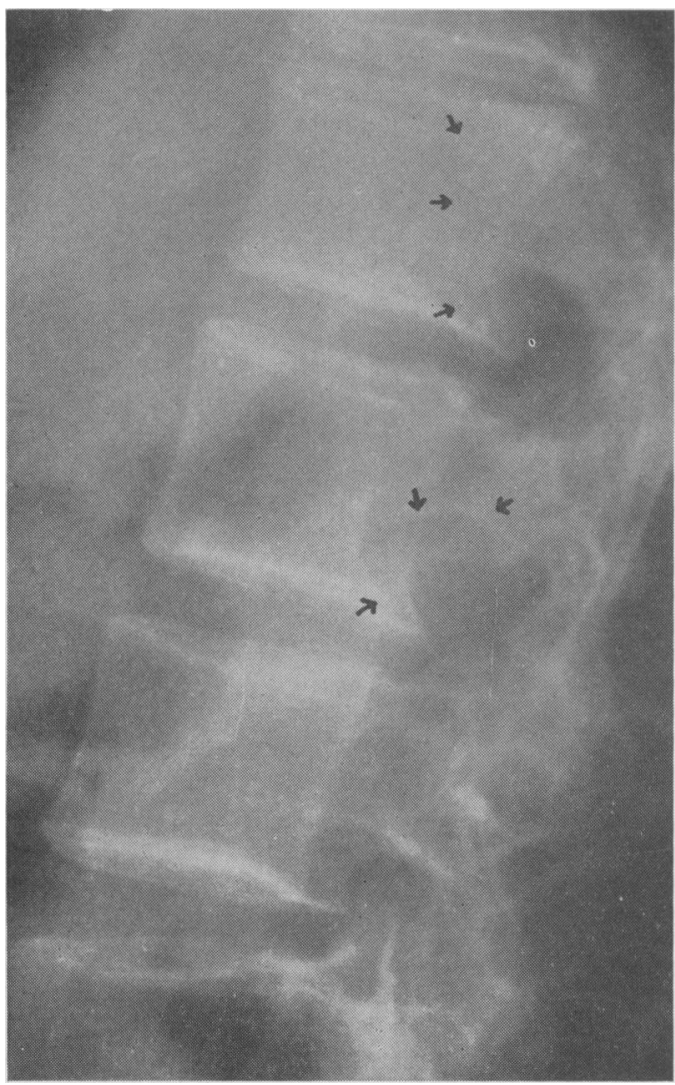

FIG. 23.-Plain lateral radiograph of the lumbar spine. Scalloping of the posterior aspects of the bodies of the upper lumbar vertebræ is shown and an enlarged intervertebral foramen is also demonstrated.

be noted that air myelography cannot show angiomas of the cord.

The value of air myelography must not be disregarded and it has received less attention outside Scandinavia than it merits. It is particularly valuable to demonstrate localized cord atrophy, whose presence should be suspected if there is an atypical clinical picture (Jakobsen and Hyllested, 1958).

\section{Cerebral Cysticercosis}

A review of 450 cases of cerebral cysticercosis revealed some interesting figures (Dixon and Lipscombe, I96I): $92 \%$ had epilepsy as the main symptom. Other symptoms were much less frequent. Mental changes occurred in only $8.7 \%$; raised intracranial pressure in $6 \%$ and cerebral lesions in $3 \% ; 3 \%$ were entirely free of symptoms. A five-to-one chance of the symptoms developing within seven years of exposure was noted, though the interval from exposure to the first symptom may be up to 30 years: $75 \%$ of cases showed radiological evidence of calcified cysts in the muscles. Calcification in the brain is less common, often inconspicuous, and occurs much later. There was only one case in which calcified intracranial cysts were present without intramuscular cyst calcification. The radiological appearances of calcified intracerebral cysts consist of very small punctate calcifications (said to be present in the scolex of the parasite) and more rarely this small, grain-like calcification may be surrounded by a halo of calcification which is present in the cyst wall (Dixon and Hargreaves, I 944). Another paper (Valopoulos and Cotsilimbas, I960) records Io cases and describes Fillipov's sign. 'This sign is shown during cerebral pneumography, when th? cysts produce filling defects in the subarachnoid spaces and in the walls of the ventricles as they protrude from the surface of the cerebral substance. Cerebral cysticercosis may also be manifested by the production of thin-walled racemose cysts which may occur in the ventricles or in the subarachnoid spaces. Hydrocephalus may be produced (Bickerstaff, Small and Woolf, I956). An annotation on this subject (Annotation, Brit. med. F., 1961) makes the observation that as world travel increases new cases may appear from time to time in Great Britain, particularly as the incidence is high in Eastern Europe and Central and Southern America.

\section{Miscellaneous Conditions}

\section{Radiographic Findings in Trigeminal Neuralgia}

Lee, in 1937, suggested an analogy between meralgia paresthetica and trigeminal neuralgia and advocated chiselling off part of the apex of the petrous bone to relieve traction on the sensory root of the fifth nerve (Lee, 1937). Basilar impression may cause elevation of the apex of the petrous bone with consequent stretching of the sensory root (Gardner, Todd and Pinto, I956). McGregor's line was used to assess the degree of basilar impression and a line through the superior borders of the orbits was used to measure the relative positions of the trigeminal impressions in the petrous bones. This method has been elaborated by Bjerrum and Thornval (1959). The figures given tend to support the hypothesis because the trigeminal impression was higher on the side with neuralgia in a significant proportion of the cases. The latter authors did not confirm the significance of basilar impression. Perhaps a more elaborate assessment of the basilar invagination, using the basal angle and Fischgold's method would have given more definite results (Bull, Nixon, Pratt and Robinson, 1959). 


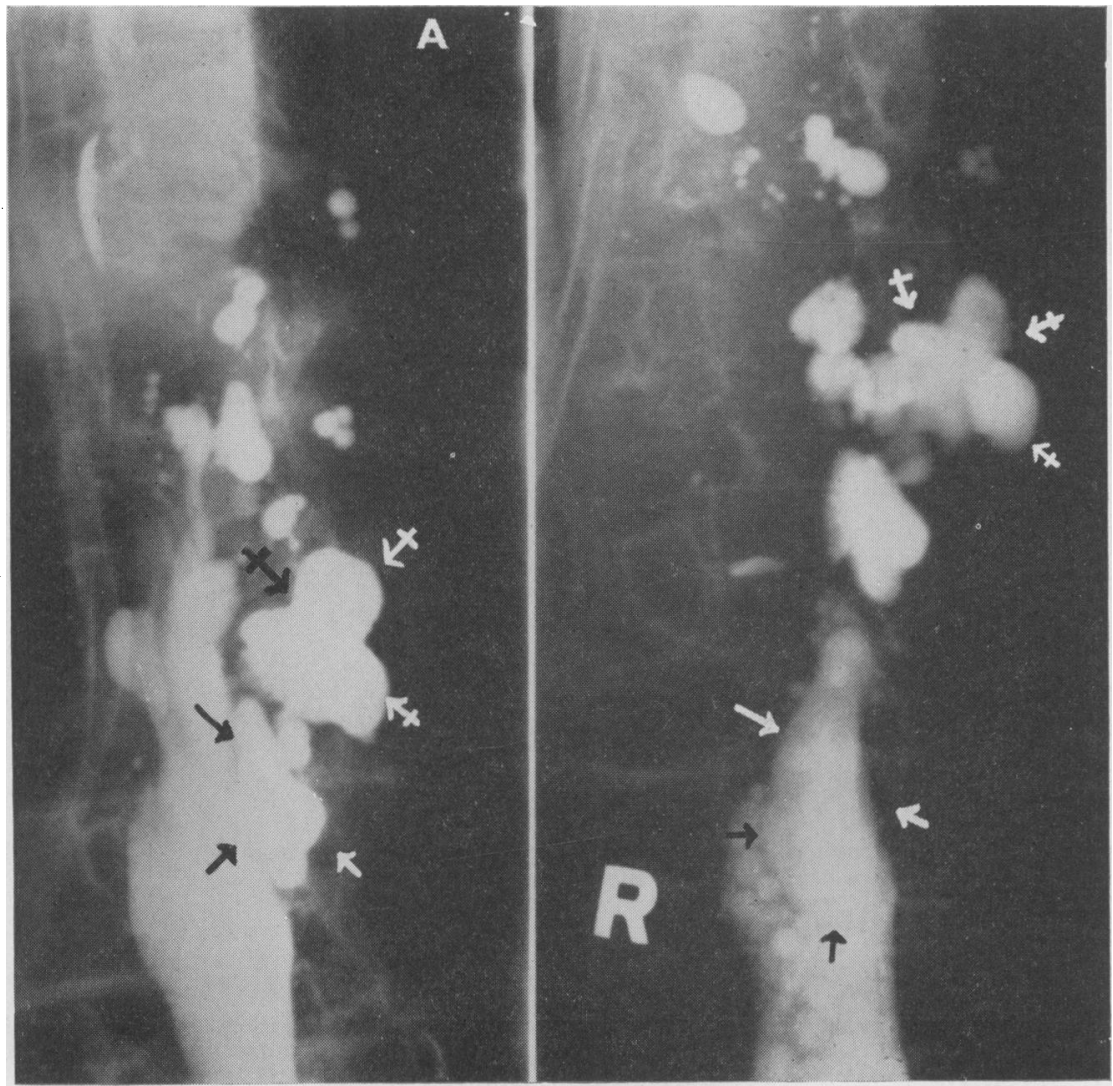

A

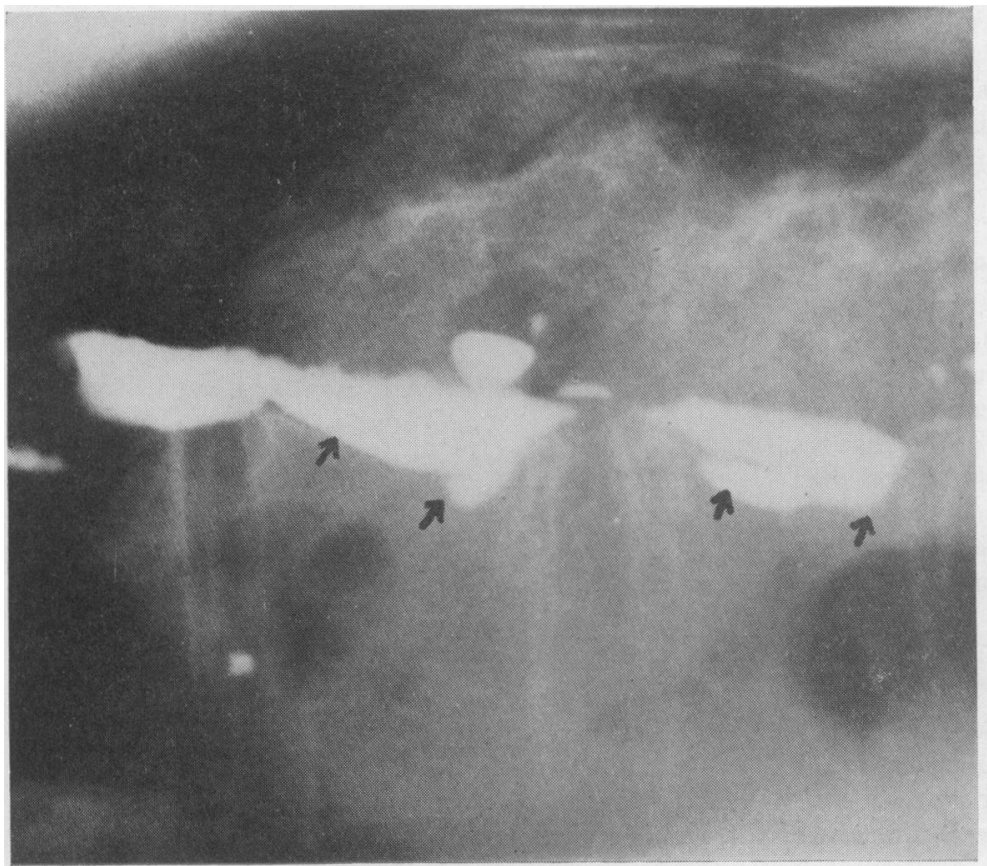

FIG. 24.-Lumbar myelogram with Myodil: (A) Numerous cysts are present filled with contrast medium. They are subarachnoid, subdural and extradural, some of the latter projecting through the intervertebral foramina. Laminectomy revealed many deficiencies in the dura through which the arachnoid matter was herniated. There was no evidence of an inflammatory process. (B) A lateral prone view showing some cysts filled with Myodil lying on the erocie 1 posterior surfaces of the bodies of the vertebræ. A normal myelographic pattern was present in the cervical region. 


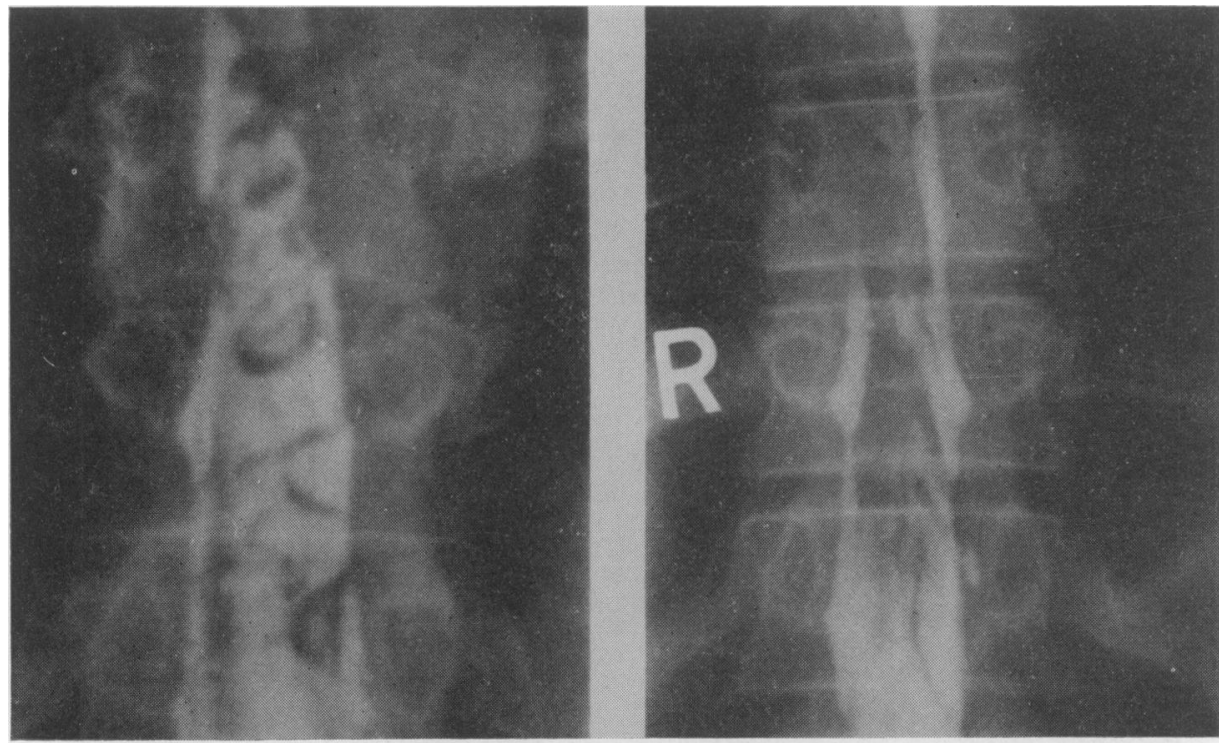

Prone

Supine

FIG. 25.-Lumbar myelogram with Myodil showing angioma of the cord hardly visible in the prone position but strikingly obvious in the supine position.

\section{Grooved Atrophy of the Parietal Bones}

Grooved or 'plate-like' areas occur in the parietal bones in old people with osteoporosis. These areas of atrophy are not to be confused with persistent parietal foramina which are congenital defects having a round and circumscribed shape and which occur at any age. Parietal emissary foramina contain an emissary vein which the other defects do not and are seldom more than $4 \mathrm{~mm}$. in size (Gros and Fortschr, 1956; Nashgold and Netsky, 1959).

\section{The Skull in Hyperparathyroid Disease}

Extensive destruction of the pituitary fossa has been described (Edeiken and Nagle, 1959) in this disease associated with extensive bone involvement elsewhere. After removal of the adenoma the sella returned to normal. A full account of the changes in the skull (Ellis and Hochstim, 1960) has shown that after removal of the parathyroid adenoma areas of destruction become recalcified with dense bone which may remain indefinitely and be mistaken for metastases.

\section{Iron-deficiency Anamias with Skull Changes}

Five patients have been described with irondeficiency anæmias in whom there were skull changes identical with those occurring in thalassemia major. All these cases were premature infants who were proved to have a purely irondeficiency state and in whom a Cooley type of anæmia had been excluded (Britton, Canby and Kohler, I960).

\section{Cranio-metaphyseal Dysplasia}

'The skull manifestations of this rare familial disorder consist of thickening of the cranial vault in the basal, frontal and occipital areas with sclerosis of the orbital ridges giving rise to a bespectacled effect. Sclerosis of the temporal bones and regions of the para-nasal sinuses areas also occurs (Schwarz, i960).

\section{The Metopic Suture}

Over 2,000 cases were examined and in $10 \%$ of female and $13.5 \%$ of males there was a persistent frontal (metopic) suture. All these cases were over 20 years of age (at which time the frontal sinuses are fully developed): $84 \%$ of these had normally-developed frontal sinuses. The authors (Marciniak and Nizankowski, I960) conclude that there is no association between absence of the frontal sinuses and the presence of a persistent frontal suture. In an examination of museum specimens of diluvial and neolithic skulls it was found that there was a higher incidence of the frontal suture in the neolithic than in the diluvial skulls and, from this fact, it is concluded that this 
is an evolutionary feature with progressive significance.

\section{Hydatid Disease of the Skull (Lipschitz, 1960)}

There are many causes of enlargement of the middle fossa. A hydatid cyst must be a very rare cause in the British Isles. Nevertheless, it is important to realize that in certain Middle Eastern countries it has been said that the commonest abdominal tumour in young people, apart from pregnancy, is a hydatid cyst, and reports of intracranial cases are increasing (Valentino, 1959). The former paper lists the plain skull changes of a middle fossa space-occupying lesion and gives the causes.

\section{Cerebral Paragonimiasis}

The practice of eating raw crustacea in some Far Eastern countries has resulted in an endemic infestation with the lung fluke Paragonimus Westermani. The common site is, of course, the lungs, but many organs may be infected and rarely the cerebral manifestations may occur first. The manifestations in the central nervous system are protean. Calcification occurs in the intracerebral cysts (Kim, I959).

\section{Osteoid Osteoma of the Frontal Bone}

This is the first case reported in the calvarium. It was confirmed by operation and showed a clear-cut, punched-out defect. Interestingly enough, it was painless (Murk, Peyser and Gellei, 1959).

\section{Basal Ganglia Calcification of the Brain (Palubinskas and Davies, 1959)}

The difficulties in understanding the patho- genesis of basal ganglia calcification have been simplified by this paper, and the relationship between the idiopathic, pseudo-hypoparathyroidism, pseudo-pseudo-hypoparathyroidism and familial basal calcification has been clarified. An informative family tree has been given, many members of which had basal ganglia calcification. Two of the cases with calcification had suggestive, though not conclusive, biochemical evidence of pseudo-hypoparathyroidism. It is reasonable to speculate that these conditions are related and various permutations may exist with formes frustes occurring. A case is described in this paper, in which the pseudo type became pseudo-pseudo when the biochemistry became normal spontaneously. During this time, however, basal ganglia calcification developed. The radial character of the basal ganglia calcification is noted and the reversed comma shape of the calcified dentate nuclei of the cerebellum is described. One of the familial cases had massive and generalized cerebral calcification with striking cortical calcification.

I am grateful to Messrs. Sierex, Elema Schonander and Messrs. Watson \& Son for the assistance and hospitality given to me. Fig. 5 has been reproduced by kind permission of the Editor of the Proceedings of the Royal Society of Medicine. I am grateful to Dr. L. O. Lanner for Fig. 6, and to Dr. Rowland Hill and Mr. Leslie Oliver for details of the case illustrated in Fig. 7; to Mr. M. Forrest for Fig. 9; to Mr. G. H. Macnab for Fig. 16; to Dr. L. Morris for Fig. 17; and to Dr. du Boulay for Fig. 18. I am also grateful to Dr. Rowland Hill and Mr. C. Westbury for the details of the case illustrated in Fig. 20 and to Dr. Meadows for Figs. 22, 24 and 25; to Miss M. Hatt-Cook for the diagrams, and to the secretarial staff of the West End Hospital for Neurology and Neurosurgery and the Westminster Hospital.

\section{REFERENCES}

Alpers, B. J., Berry, R. G., and Paddison, R. M. (1959): Anatomical Studies of the Circle of Willis in Normal Brain, Arch. Neurol. (Chicago), 8I, 409.

Andersen, P. E. (1958): The Lentriculostriate Arteries and Their Diagnostic Value-a preliminary report, Acta radiol. (Stockh.), 50, 84.

AnNotation (1961): Cysticercosis, Brit. med. ., i, 804.

Azambuja, N., Lindgren, E., and Sjögren, S. E. (1956): Tentorial Herniation, Anatomy, Pneumography and Angiocardiography, Acta radiol. (Stockh.), 46, 215.

Barbieri, P. L., and Verdecchia, G. C. (I957): Vertebral Arteriography by Percutaneous Puncture of the Subclavian Artery, Ibid., 48, 444 .

Baker, A. H. (196I): Symposium on the Surgical Treatment of Extracranial Occlusive Cerebro-vascular Disease, Proc. Mayo Clinic, 35, 17.

De Bakey, M. E., and Crawford, E. S. (1958): Surgical Treatment of Basilar Artery Insufficiency, f. Amer. med. Ass., 168, 509 .

BEGG, A. C. (1960): Some Radiologic Aspects of Ischæmia of the Brain, Brit. F. Radiol., 33, 398.

BERK, M. E. (196r): Combined Carotid Vertebral Angiography: A Method of Vertebral Arteriography, Ibid., $33,780$.

Bickerstaff, E. R., Small, J. M., and Woolf, A. L. (1956): Cysticercosis of the Posterior Fossa, Brain, 79, 622.

BJerrum, J., and Thornval, G. (1959): Roentgenographic Findings in Trigeminal Neuralgia, Acta radiol. (Stockh.), $5 \mathrm{I}, \mathbf{2 8 9}$.

Du Boulay, G. (r96r): Personal communication.

Britton, H. A., Canby, J. P., and Kohler, C. M. (1960): Iron Deficiency Anæmia Producing Evidence of Marrow Hyperplasia in the Calvarium, Pediatrics, 25, 261.

BucY, P. C. (1950): In Brennerman's 'Practice of Pediatrics', chap. 3. Hagerstown: Prior.

Bull, J. W. D., Marshall, J., and Shaw, D. A. (1960): Cerebral Angiography in the Diagnosis of the Acute Stroke, Lancet, i, 562. 
Nixon, W. L. B., Pratr, R. T. C., and Robinson, R. K. (1959): Paget's Disease of the Skull and Secondary?

Basilar Impression, Brain, 82, 10.
Crofton, F. D. L., and Matson, D. D. (1960): Roentgenoligic Study of Choroid Plexus Papillomas in Childhood Amer. Y. Roentgenol., 84, 479.

Cronevist, T. S. (1959): Myelography After Operation for Herniated Lumbar Discs, Acta radiol. (Stockh.), 52, 45. Davies, H. W. (1951): Positive Contrast Myelography, Proc. roy. Soc. Med., 44, 881.

Dixon, H. W., and LIPSCOMBe, F. M. (1961): ‘Medical Research Council Report on Cysticercosis'. London: H.M.S.O , and Hargreaves, W. H. (1944): Cysticercosis: A Further Ten-year Clinical Study, Quart. $\mathcal{F}_{\text {. Med., 13, 107. }}$

Donaldson, A. A., and GillinghaM, F. G. (1960): Radiology in Stereotaxic Surgery, Brit. F. Radiol., 137, 757.

EDEIKEN, J., and NAGLE, W. (1959): Hyperparathyroidism with Pseudo-erosion of the Sella: Report of a Case, Arch등 intern. Med., I04, 430 .

Elfin, P. (1960): Angiography of the Carotid Artery with the Use of Catheter Technique, Radiology, 75, 80.

Elkington, J. ST. C. (1958): Cerebrovascular Disease in the Light of Modern Techniques, Lancet, ii, 275.

Eulis, K., and Hochstrm, R. J. (1960): The Skull in Hyperparathyroid Disease, Amer. Y. Roentgenol., 83, 733.

FORREST, M., LAURENCE, R. M., and MCNAB, G. H. (1957): Ventriculo-subdural Drainage in Infantile Hydrocephalus! Analysis of Early Results, Lancet, ii, 1274 .

FredZell, G., and LindGren, E. (1960): Mimer, Acta radiol. (Stockh.), 5, 209.

GARDNER, W. J., TODD, E. M., and PINTO, J. P. (1956): Roentgenographic Findings in Trigeminal Neuralgia, Amer F. Roentgenol., 76, 346.

GensinI, G. G., and ECKER, H. (1960): Percutaneous Aortocerebral Angiography, Radiology, 2, 855 .

GreITZ, T. (1956): A Radiologic Study of the Brain Circulation by Rapid Serial Angiography of the Carotid Artery Acta radiol. (Stockh.), Suppl. 140.

Gros, J., and ForTSCHR, A. D. (1956): Grooved Atrophy of the Parietal Bones, Fortschr. Röntgenstr., 85, 154.

Hobss, H. E., Du Boulay, G., and Davis, R. E. (1960): Orbital Angioma Diagnosed by Phlebography, Brit. Y F Ophthal., 44, $55 \mathrm{r}$.

Hutchinson, J. R., and Yates, P. O. (1957): Carotico-vertebral Stenosis, Lancet, i, 2.

JAKOBSEn, H. E., and Hyllested, K. (1958): Localized Atrophy of the Spinal Cord, Acta radiol. (Stockh.), 58m, 2110

JING, B. S., and SCHECHTER, M. M. (1960): Improved Visualization of the Ventricular System with the Technique of Automography, Radiology, 74, 593 .

Jrrout, J. J. (1959a): The Mobility of the Cervical Spine Under Normal Condition, Brit. F. Radiol., 32, 744.

(r959b): Myelographic Syndrome of Caudal Dislocation of the Brain Stem, Ibid., 32, 188.

KIM, S. K. (1959): Cerebral Paragonimiasis, A.M.A. Arch. Neurol. Psychiat., r, 30.

Kraus, E. (1958): Encephalographic Sign in Pineal Tumours, Acta radiol. (Stockh.), 50, 12.

KLEFENBERG, G., and SaltzMan, G. F. (1959): Gas Myelography Studies in Syringomyelia, Ibid., 52, 129.

KRAYENBUHL, H., and YASARGIL, M. C. (1957): 'Die vasculasen Evkrankungen im Gebeit des arterie vertebralis arterie basilaris'. Stuttgart: Georg Thieme Verlag. (1958): Diagnostic Value of Orbital Angiography, Brit. Y. Ophthal., 42, 180.

KuHN, R. A. (1960): Brachial Cerebral Angiography, $\mathcal{F}$. Neurosurg., 27, 955. (1959): Importance of Accurate Diagnosis by Cerebral Angiography in Cases of 'Stroke', f. Amer. med. Ass. I69, 1867.

LANNER, L. O. (1961): Personal communication.

Lawrence, K. M. (1960): The Natural History of Hydrocephalus, Postgrad. med. F., 36, 662.

LEE, F. C. (1937): Trigeminal Neuralgia, F. med. Ass. Ga, 26, 432.

LILIEQUIST, B. (1959a): The Subarachnoid Cisterns: An Anatomic and Roentgenologic Study, Acta radiol. (Stockh.) Suppl. 185 .

- (1959b): Pontine Angle Tumour: Encephalographic Appearances, Ibid., Suppl. I86.

- (1960): Encephalography in the Arnold-Chiari Malformation, Ibid., 53, 17. psychiat. scand., 35, 223.

LinDGREN, E. (1957): Radiologic Examination of the Brain and Spinal Cord, Acta radiol. (Stockh.), Suppl. I $5 \mathrm{I}$.

(1956): Another Method of Vertebral Arteriography, Ibid., 46, 257.

LIPSCHITZ, R. (1960): Asymmetry of Skull Due to Hydatid Disease, Brit. F. Radiol., 33, 397.

Lombardi, G., and Migliacavva, F. (1959): Angiomas of the Spinal Cord, Ibid., 32, 810.

McKissock, W., Richardson, A., and Walsh, L. (1959): Surgery of Primary Intracerebral Hæmorrhage, Lanceto ii, 683 .

-, and PAINE, K. W. E. (1959): Subarachnoid Hæmorrhage, Brain, 82, 356.

-, Crawford, T., Bull, J. W. D., and PerRetT, L. V. (1959): Some Aspects of Subarachnoid Hæmorrhage: Symposium, Brit. Y. Radiol., 32, 79.

MacNab, G. H. (1959): The Spitz-Holter Valve, F. Neurol., Neurosurg. Psychiat., 22, 82.

MAHONEY, G. G. DE G. (1960): Autotomography, Ibid., 27, 81. MAHONEY, G. G. DE G. (1960): Autotomography, Ibid., 27, 81.
MARCINIAK, R., and NiZANKowSKI, C. (I960): Metopism and its Correlation with the Development of the FrontaP
Sinuses, Acta radiol. (Stockh.), 5I, 343.

MoRRIS, L. (1959): Angiographic Demonstration of the Vertebral Arteries with Special Reference to Percutaneou河 Subclavian Puncture, Brit. F. Radiol., 42, 673 .

(1960): Angioma of Cervical Spinal Cord, Radiology, 75, 785.

(196I): Personal communication.

Mulvey, R. B. (1960): An Unusual Pattern of Arachnoiditis, Radiology, 75, 778.

MuRK, J., PEYSER, E., and Gellei, B. (1959): Osteoid Osteoma of the Frontal Bone, Brit. Y. Radiol., 33, 389.

MURPHY, F., and SHILlITo, J. (1959): Avoidance of False Angiographic Localization of the Site of Internal Carotid Stenosis, Y. Neurosurg., 16, 24.

MurRay, R. O. (1959): Subdural Arachnoid Cyst of Lumbar Spinal Canal, Brit. Y. Radiol., 32, 589. 
Nashgold, B. S., Jr., and Netsky, M. G. (1959): Foramens Fenestræ and Thinness of the Parietal Bone, F. Neuropath. exp. Neurol., 18, 432.

Newton, T. H., and Couch, R. S. C. (1960): Internal Carotid Occlusion: Possible Errors in the Arteriographic Diagnosis of, Radiology, 75, 766 .

Oliver, L. C. (1958): Primary Arachnoid Cysts, Brit. med: F., i, I 147.

Palubinskas, A. J., and Davies, H. W. (1959): Calcification of the Basal Ganglia of the Brain, Amer. F. Roentgenol., 82, 806.

Pygott, F., and Hutton, C. F. (1959): Vertebral Arteriography by Percutaneous Brachial Artery Catheterization, Brit. F. Radiol., 32, I14.

RoB, C., and WheELER, E. B. (1957): Surgery of the Cerebral Vessels in the Neck, Brit. med. F., ii, 264.

RugGiEro, G., and JAY, M. (1958): Une technique pour l'Artériographie de l'Artière Carotide Extrême, Acta radiol. (Stockh.), 50, 453.

Saltzman, G. F. (1959a): Circulation Through the Anterior Communicating Artery by Carotid Arteriography, Ibid., 51, 194 .

- (1959b): Circulation Through the Posterior Communicating Artery in Different Compression Tests, Ibid., 5I, 10.

- (1959c): Infundibular Widening of the Posterior Communicating Artery Studied by Carotid Arteriography, Ibid., 5I, 415.

Schechter, M. M., Bull, J. W. D., and Carey, P. (1958): Two New Encephalographic Signs of Pressure Hydrocephalus, Brit. F. Radiol., 31, 3 I7.

Schmitz, A. L. (1959): Changes in Ambient Wing Cisterns Secondary to Intracranial Masses, Acta radiol. (Stockh.), 52, 209.

SchuARz, J. (1959): Autotomography of the Fourth Ventricle and Floor of the Third Ventricle, Ibid., 52, 465:

Schwarz, E. (I960): Cranio-metaphyseal Dysplasis, Amer. F. Roentgenol., 84, 46r.

Sheldon, P. A. W. (1956): A Special Needle for Percutaneous Vertebral Angiography, Brit. F. Radiol., 29, 231.

SimON, M. (I956): Personal communication.

Sitz, E. R. (1959): Neurosurgery in Prevention of Exogenous Mental Retardation, Pediat. Clin. N. Amer., 6, 1215.

Sutron, D. (1950): Anomalous Carotid-basilar Anastomosis, Brit. F. Radiol., 23, 6r7.

Swann, G. F. (1957): Based on a paper read at the Annual Congress of the British Institute of Radiology, 1957. Quoted by Pribram, H. W. F., and Swann, G. F. (1960): Incidence of Endocrine Effects in Sellar and Parasellar Tumours, Radiology, 2, 877.

(1960): Technique of Positive Contrast Myelography, Proc. roy. Soc. Med., 53, 448.

- (1958): Vertebral Arteriography Using the Sheldon Needle and Modifications of It, Brit. F. Radiol., 31, 23.

(1961): In press.

Teng, P. (1960): Myelographic Identification of Dentate Ligaments, Radiology, 74, 944.

VALENTINo, V. (1959): Combined Intraventricular and Intracerebral Hydatid Cysts, Radiology, 73, 250.

Vallebona, A. (193I): Una Modalita di Tecnica per la dissociazione Radiographica delle ombre applicate all studio-del cranio, Radiol. med. (Torino), 17, 340.

Valopoulos, C., and Cotsilimbras, D. (1960): On Ten Cases of Cysticercosis, Evangelismos Hosp. Rev., 22, 645.

WICKBOM, I. (196I): Personal communication.

Weidenman, O., and ForTsChr, G. E. B. (1960): Method for Demonstration of Upper Thoracic Spinal Cord in Positive Myelography, Fortschr. Röntgenstr., 92, 170.

Zellweger, H., and VAN EPPS, E. F. (1959): The Cavum Veli Interpositi and its Differentiation From the Cavum Vergæ, Amer. Y. Roentgenol., 82, 793.

Ziedses Des Palantes, B. G. (I950): Examen du troisième et du quatrième ventricule au moyen de petites quantités d'air, Acta radiol. (Stockh.), 34, 399.

(1956): Personal communication. 